\title{
Development of quantitative structure gas chromatographic relative retention time models on seven stationary phases for 209 polybrominated diphenyl ether congeners
}

\author{
Yawei Wang ${ }^{\mathrm{a}}$, An $\mathrm{Li}^{\mathrm{b}}$, Hanxia Liu ${ }^{\mathrm{a}}$, Qinghua Zhang ${ }^{\mathrm{a}}$, \\ Weiping $\mathrm{Ma}^{\mathrm{c}}$, Wenlu Song ${ }^{\mathrm{b}}$, Guibin Jiang ${ }^{\mathrm{a}, *}$ \\ ${ }^{a}$ State Key Laboratory of Environmental Chemistry and Ecotoxicology, Research Center for Eco-Environmental Sciences, \\ Chinese Academy of Sciences, P.O. Box 2871, Beijing 100085, China \\ ${ }^{\mathrm{b}}$ School of Public Health, University of Illinois at Chicago, 2121 West Taylor Street, MC-922, Chicago, IL 60612-7260, USA \\ ${ }^{\mathrm{c}}$ Department of Chemistry, Lanzhou University, Lanzhou 730000, China
}

Received 14 September 2005; received in revised form 9 November 2005; accepted 11 November 2005

Available online 13 December 2005

\begin{abstract}
Quantitative structure retention relationships (QSRRs) were developed to predict the gas chromatographic (GC) relative retention times (RRTs) for 209 polybrominated diphenyl ether (PBDE) congeners using the heuristic method included in the computer software Comprehensive Descriptors for Structural and Statistical Analysis (CODESSA). A total of 445 constitutional, topological, geometrical, electrostatic, and semi-empirical quantum chemical descriptors were derived for all PBDEs. Using experimental RRT data for 126 PBDE congeners from the literature, predictive regression models were built for seven individual GC capillary columns differing in stationary phases. Each model includes four descriptors which included Wiener index, Randic index, polarity parameter, etc., selected by CODESSA. High predictability was obtained. High multiple correlation coefficients $R^{2}$ indicated that $>98.5 \%$ (except for stationary phase CP-Sil 19) of the total variation in the predicted RRTs is explained by the fitted models. The models were subsequently used to predict the RRTs of the remaining 83 PBDE congeners on seven different stationary phases. The statistical results show that, compared with others, DB-XLB column not only produces the least number of peak overlaps but also results in shorter retention times.
\end{abstract}

(C) 2005 Elsevier B.V. All rights reserved.

Keywords: Polybrominated diphenyl ethers; Quantitative structure retention relationships; Prediction; Gas chromatography; Relative retention time; Stationary phases

\section{Introduction}

Polybrominated diphenyl ethers (PBDEs) are a group of brominated flame retardants (BFRs), which have been manufactured in large quantities and widely used in a variety of consumer goods [1]. They spread ubiquitously as environmental contaminants and have been detected in a wide range of environmental samples and humans [2-5]. It is known that PBDEs are persistent, bioaccumulative, endocrine disrupting, and possibly carcinogenic [6].

\footnotetext{
* Corresponding author. Tel.: +86106284 9334; fax: +861062849179.

E-mail address: gbjiang@ rcees.ac.cn (G. Jiang).
}

Similar to polychlorinated biphenyls (PCBs), there are 209 possible PBDE congeners, differing in the number and positions of bromine atoms in the molecule. Unlike PCBs, however, only about a dozen congeners exist as major components in the commercial PBDEs mixtures [7]. Due to this fact and the lack of commercially available analytical standards, many previous PBDE studies have targeted on a limited number of congeners. However, a number of recent studies indicate that degradations of highly brominated PBDEs, such as the most heavily manufactured decabromodiphenyl ether, can lead to a variety of less brominated congeners $[8,9]$. The congener patterns of the degradation products are often complex, containing numerous congeners which may not exist in the PBDE technical mixtures. Accurate identification of these reaction products is essential in understanding the pathways and mechanisms of PBDE degra- 
dation processes, which determine their persistence and fate in the environment.

Analyses of PBDEs in environmental samples use highresolution gas chromatography (HRGC) almost exclusively, coupled with appropriate detectors including electron-impact (EI) mass spectrometer (MS), electron-capture negative chemical ionization (NCI) MS or others [10-12]. Identification of the PBDE congeners in unknown samples relies on the matching of characteristic MS spectrum and/or the GC retention time with those of pure chemical standards. However, such efforts are often prohibited by the lack and/or the costs of the chemical standards. Predictive tools are needed to assist researchers in identifying PBDEs for which chemical standards are not available.

Quantitative structure retention relationships (QSRRs) describe the effects of an analyte's molecular structure on chromatographic retention time and also explore the mechanisms of absorption in stationary phase and elution from the column [13]. QSRRs have been developed for a number of halogenated organic contaminant groups [14-17]. For PBDEs, Rayne and Ikonomou presented several predictive equations with five or seven simple parameters, including the numbers of ortho, meta, and para substituents, as well as molecular mass and calculated ionization potential and dipole moment, as independent variables [18-20]. The reliability of equations was examined by using basic statistics such as $R^{2}$ and $F$ values rather than the predictions of relative retention times (RRTs) for PBDE congeners, which were not involved in model development. Also, their studies are limited to only one type of GC stationary phase (DB-5 or DB-5HT).

The purpose of this work was to characterize PBDE chromatographic retention properties on various stationary phases using topological and other molecular descriptors. A retentiontime database obtained by Korytár et al. [21] was used to derive a series of QSRR models based on the heuristic method of Comprehensive Descriptors for Structural and Statistical Analysis (CODESSA) technique. The models were evaluated by predicting the GC-RRTs of PBDE congeners in a test set that was included in the database but independent of model development. Then, the experimental GC-RRTs of selected PBDEs (BDE 17, $28,47,66,71,85,99,100,138,153,154,183,190$, and 209) obtained in our laboratories are compared to the reference data and predicted values.

\section{Methods}

\subsection{Chemicals and GC systems}

Two sets of gas chromatographic (GC) retention times for PBDEs were obtained from our laboratories using different instruments and operational conditions. One of the analyses involved 14 PBDE congeners, including BDE 17, 28, 47, 66, $71,85,99,100,138,153,154,183,190$, and 209. The PBDE analytical standards were purchased from Cambridge Isotope Laboratory (Andover, MA). The analysis was performed on an Agilent 6890 GC coupled with a Waters Autospec Ultima high-resolution mass spectrometer (HRMS) (Waters Micromass, Manchester, UK) operating at $35 \mathrm{eV}$ and the trap current was $600 \mu \mathrm{A}$. The GC was equipped with a CTC PAL autosampler. One microliter of sample was injected in splitless mode in a DB-5MS fused silica capillary column $(12 \mathrm{~m} \times 250 \mu \mathrm{m}$ i.d. $\times 0.25 \mu \mathrm{m}$ film thickness) with helium as carrier gas at a constant flow rate of $1.0 \mathrm{ml} \mathrm{min}{ }^{-1}$. The initial oven temperature was $110^{\circ} \mathrm{C}$, which lasted for $3 \mathrm{~min}$. The oven temperature was increased to $285^{\circ} \mathrm{C}$ at $15^{\circ} \mathrm{C} \mathrm{min}^{-1}$, held for $4 \mathrm{~min}$, and then increased to $325^{\circ} \mathrm{C}$ at $10^{\circ} \mathrm{C} \mathrm{min}^{-1}$, and held for $10 \mathrm{~min}$.

The other laboratory work used nine PBDE congeners, including BDEs 28, 47, 66, 85, 99, 100, 153, 154, and 183. Separation was performed on an Agilent Model 6890 GC coupled with a Model 5973 low-resolution mass spectrometer (LRMS). Samples were introduced into the GC through a programmable temperature vaporization (PTV) large volume injection port. The temperature program for the PTV port was from $0{ }^{\circ} \mathrm{C}$ (held for $1.5 \mathrm{~min}$ ) to $300^{\circ} \mathrm{C}$ at $600^{\circ} \mathrm{C} \mathrm{min}^{-1}$. A DB-5MS ( $30 \mathrm{~m} \times 0.25 \mathrm{~mm}$ i.d., $0.25 \mu \mathrm{m}$ film thickness; J\&W Scientific) capillary column was used for separation, with helium as the carrier gas at a flow rate of $1 \mathrm{ml} \mathrm{min}{ }^{-1}$. The initial oven temperature was $140{ }^{\circ} \mathrm{C}$, which was held for $3 \mathrm{~min}$, increased to $200^{\circ} \mathrm{C}$ at $15^{\circ} \mathrm{C} \mathrm{min}{ }^{-1}$, increased to $220^{\circ} \mathrm{C}$ at $2{ }^{\circ} \mathrm{C} \mathrm{min}^{-1}$, increased to $315^{\circ} \mathrm{C}$ at $5^{\circ} \mathrm{C} \mathrm{min}^{-1}$, and finally held for $5 \mathrm{~min}$ at $315^{\circ} \mathrm{C}$. The electron impact (EI) MS was operated in selected ion monitoring (SIM) mode.

\subsection{Relative retention database for PBDEs}

An experimental reference GC-RRT database was built by Korytár et al. [21]. The database contains RRTs for 126 PBDE congeners on seven GC columns of different stationary phases including DB-1 (100\% dimethylpolysiloxane), DB-5 (5\% phenyl $95 \%$ dimethyl arylene siloxane), HT-5 (5\% phenyl siloxane-carborane), DB-17 (50\% phenyl 50\% dimethyl arylene siloxane), DB-XLB (proprietary phase), HT-8 (8\% phenyl-polycarboranesiloxane), and CP-Sil 19 (14\% cyanopropyl-phenyl, 86\% dimethyl polysiloxane). All experiments were carried out with an Agilent 6890 GC equipped with an electron capture detector (ECD). The RRTs were calculated by dividing the obtained retention times by the sum of the retention times for 2,2',4,4' -tetrabrominated diphenyl ether (BDE47)

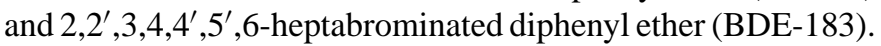

In this work, this reference database was divided into two parts. A total of 100 PBDE congeners in the database formed a "training set" for model development, and the other 26 congeners were used as a "test set". These 26 congeners were randomly chosen, with the number of bromines ranging from 2 to 8 .

\subsection{Model development using CODESSA}

In this work, chemical structures of 209 individual PBDE congeners were drawn and modeled using the MM+ method of HyperChem Release 7.0 software (Hypercube, 2002). Optimization of the chemical structures was performed with the semiempirical AM1 method in HyperChem Release 7.0 software and saved as hin files for MOPAC [22]. Then, the hin files were transferred into CODESSA $[23,24]$ software program to calculate a set of 445 constitutional, topological, geometrical, 
electrostatic, and semi-empirical quantum chemical descriptors for each of the 209 PBDE congeners.

One of the computational procedures built in CODESSA uses the heuristic method. The advantages of the heuristic method are the high speed of computation and the absence of restrictions on the size of the data set. The heuristic method proceeds with a preselection of descriptors by sequential elimination of descriptors, which do not meet any of the following criteria: (i) the $F$-test greater than one unit; (ii) $R^{2}$ value less than a value defined at the start (default 0.01); (iii) the Student's $t$-test less than that defined (default 0.1); and (iv) duplicate descriptors having a higher squared inter-correlation coefficient than a predetermined level (usually 0.8).

The goodness of the correlation was examined by the correlation coefficient $\left(R^{2}\right)$, the $F$-test $(F)$, and the squared standard error $\left(s^{2}\right)$. The stability of the models was evaluated against the cross-validated coefficient, $R_{\mathrm{cv}}^{2}$, which describes the stability of an obtained regression equation by focusing on the sensitivity of the model to the elimination of any single data point.

\section{Results}

By the heuristic method, regression equations were obtained using the GC-RRTs of the 100 BDE congeners in the training set for seven different stationary phases. The heuristic correlations performed provided the optimal equations for different numbers of descriptors in the range of $1-6$. Plots of $R^{2}$ and $R_{\mathrm{cv}}^{2}$ values against the number of descriptors (Fig. 1), which provided guidance in deciding the number of descriptors to be retained in the models, suggested that effectual models included four descriptors. The abbreviations of the descriptors are listed in Table 1 and the resulting regression equations for individual GC stationary phases are summarized in Table 2 . The reference and predicted GC-RRT values for the training set on seven stationary phases are given in Table 3 .

The multiple correlation coefficients $\left(R^{2}\right)$ are higher than 0.985 for all GC stationary phases except CP-Sil 19 (Table 2). The overall $F$-values of $>700$ and the PRESS (predicted error

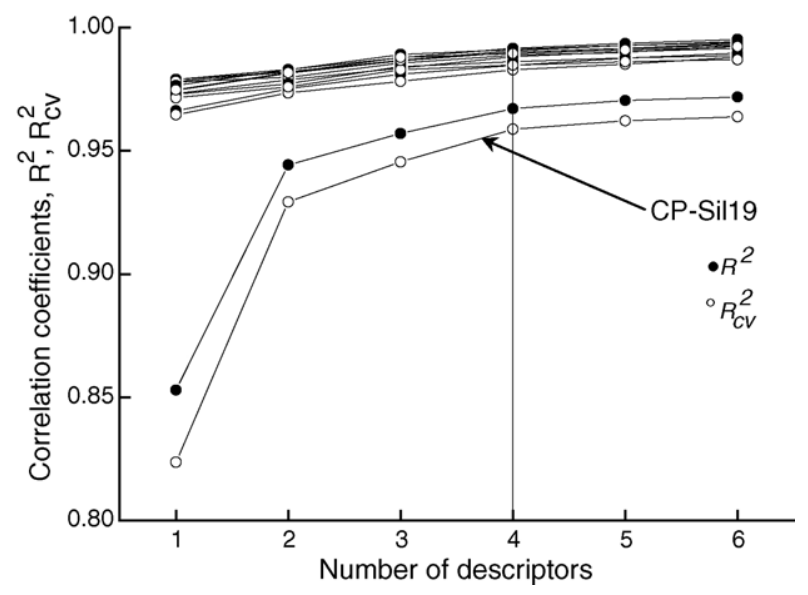

Fig. 1. Correlation coefficients $\left(R^{2}, R_{\mathrm{cv}}^{2}\right)$ (seven models) vs. number of descriptors.
Table 1

The abbreviation of the descriptors' name

\begin{tabular}{|c|c|c|}
\hline & Descriptor & Abbreviate \\
\hline 1 & Wiener index ${ }^{\mathrm{a}}$ & $W$ \\
\hline 2 & $\begin{array}{l}\text { Complementary information content } \\
(\text { order } 0)^{\mathrm{a}}\end{array}$ & $C$ \\
\hline 3 & $\begin{array}{l}\text { Maximum partial charge for a } \mathrm{Br} \text { atom } \\
{[\text { Zefirov's PC }]^{\mathrm{b}}}\end{array}$ & $\operatorname{Max}_{\mathrm{Br}}$ \\
\hline 4 & $\begin{array}{l}\text { Minimum electrophilic reaction index } \\
\text { for a } \mathrm{O} \text { atom }\end{array}$ & $\operatorname{Min}_{O}$ \\
\hline 5 & Principal moment of inertia $\mathrm{A}^{\mathrm{d}}$ & PA \\
\hline 6 & XY shadow/XY rectangle ${ }^{\mathrm{d}}$ & $X_{\text {Shadow }}$ \\
\hline 7 & Randic index $(\text { order } 3)^{\mathrm{a}}$ & $R$ \\
\hline 8 & $\begin{array}{l}\text { RPCG relative positive charge } \\
\text { (QMPOS/QTPLUS) [Zefirov's PC] }\end{array}$ & RPCG \\
\hline 9 & $\begin{array}{l}\text { Min partial charge for a } \mathrm{C} \text { atom } \\
{[\text { Zefirov's } \mathrm{PC}]^{\mathrm{b}}}\end{array}$ & $\operatorname{Min}_{C}$ \\
\hline 10 & Polarity parameter $\left(Q_{\max }-Q_{\min }\right)^{\mathrm{b}}$ & $P$ \\
\hline 11 & HOMO-1 energy ${ }^{c}$ & HOMO-1 \\
\hline 12 & Moment of inertia $C^{d}$ & $\mathrm{MC}$ \\
\hline
\end{tabular}

sum of squares) of $<0.00074$ (except for CP-Sil 19, Table 3) demonstrate that the regressions are highly significant.

The results in Table 2 indicate that GC-RRTs of PBDEs on the four stationary phases DB-1, DB-5, HT-5, and DB-17 are strongly dependent on the Wiener index [25], the first topological index used in chemistry [26]. It is the sum of distances between all the pairs of vertices in a hydrogen-suppressed molecular graph. The coefficient of correlation between the GC-RRTs of PBDEs and Wiener index was 0.987, 0.989, 0.983, and 0.986, respectively, for the four stationary phases. GC-RRTs of PBDEs on the stationary phases DB-XLB, HT-8, and CP-Sil 19 are dependent strongly on the third order simple Randic index, an adjacency-based topological index proposed by Randic [27], with the correlation coefficients being $0.983,0.982$, and 0.891 , respectively.

The RRTs of 26 BDE congeners in the test sets were obtained using the models presented in Table 2 . Good predictive ability was demonstrated by the results, as shown in Fig. 2. For all stationary phases with the exception of CP-Sil 19, the regressions of predicted RRTs versus reference RRTs have slopes close to unity $(0.995-1.104) . R^{2}$ ranges from 0.985 to 0.996 and is not statistically different from those reported in Table 2 for the training sets for each of the seven stationary phases.

\section{Discussions}

\subsection{The major contributing molecular descriptors}

The correlations between RRTs of organic compounds and Wiener index or Randic index (order 3) show that the magnitude of intermolecular interactions between the compounds and the stationary phase is related to the degree of branching of the molecules. For example, Wang et al. [28] showed that there were significant relationships between retention indices of saturated 
esters on seven stationary phases of different polarity and the Wiener index or Randic index. For PBDEs as well as similarly structured polyhalogenated aromatics such as PCBs, it is not difficult to understand the relationships. The Wiener index that represents molecular "steric-branching" increases as the number of halogen substitution increases since it is defined as the halfsum of all topological distances in the hydrogen-depleted graph representing the skeleton of the molecule.

Within a PBDE homolog, the positions of bromines determine the optimal molecular conformation and affect the GC retention. The differences in RRTs among structural isomers may not be fully accounted for by either Wiener or Randic index alone although plots of reference RRTs [21] versus either of these indices alone result in positive slopes in most cases. It is noticed that Wiener index tends to increase with increasing number of para bromines and to decrease with increasing ortho bromines, within individual homologs. The Wiener index also tends to be lower if one of the two phenyl rings is fully brominated or if all bromines are on the same ring.

The descriptor "maximal partial charge for a $\mathrm{Br}$ atom [Zefirov's PC]" reflects the characteristics of the charge distribution of a molecule; "minimum electrophilic reaction index for an oxygen atom" estimates the relative reactivity of the atoms in the molecule for a given series of compounds and are related to the activation energy of the corresponding chemical reaction. For PBDEs with similar structures, such as those having the same number of bromine, these factors will play important roles in deciding the elution order on columns.

\subsection{Comparison between RRT prediction approaches}

For halogenated aromatic compounds, various approaches of RRT prediction have been proposed. Ong and Hites [14] introduced linear predictive equations to investigate the relationships between three physical properties (molecular polarizabilities, ionization potentials, and dipole moments) and RTs of three groups of compounds (PAH, PCB, PCDF). They found molecular polarizability was the most important variable. Rayne et al. [20] reported a gas chromatographic relative time model, which uses molecular descriptors including the number of ortho-, meta, and para-bromine substituents, the calculated dipole moment, and the natural logarithm of molecular weight, to predict GC retention times of the 163 individual PBDE congeners by using GC-RRTs of 46 available PBDEs on DB-5 stationary phase. The resulting model is:

$$
\begin{aligned}
\mathrm{RRT}= & (-6.597 \pm 0.253)+(-0.076 \pm 0.007)(\text { no. } o-\mathrm{Br}) \\
& +(-0.041 \pm 0.007)(\text { no. } m-\mathrm{Br}) \\
& +(-0.021 \pm 0.011)(\text { no. } p-\mathrm{Br})+(0.012 \pm 0.006)(\mu) \\
& +(1.247 \pm 0.046)(\ln \mathrm{MW}), \\
R^{2}= & 0.997, \quad F=1137.2, \quad n=46
\end{aligned}
$$

An apparent advantage of this type of approach is the use of structural parameters that have straightforward physical meanings and are familiar to most analytical chemists. Our study, to our knowledge, is the first one using combinations of con- 
Table 3

Elution order of $100 \mathrm{BDE}$ congeners (training set), $26 \mathrm{BDE}$ congeners (test set) and predicted set (83 BDE congeners) on seven capillary columns and their ref. [21] and predicted GC-RRT

\begin{tabular}{|c|c|c|c|c|c|c|}
\hline DB-1 & DB-5 & HT-5 & DB-17 & DB-XLB & HT- 8 & CP-Sil 19 \\
\hline PRE & PRE REI & PRE REF & PRE REF & DE PRE REF & DE PRE RE & DE PRE RE \\
\hline 10.0820 .100 & 10.0340 .095 & \begin{tabular}{l|ll|}
2 & 0.046 & 0.091
\end{tabular} & \begin{tabular}{l|lll}
2 & 0.039 & 0.086
\end{tabular} & 10.0350 .086 & $\begin{array}{llll}1 & 0.039 & 0.084\end{array}$ & \begin{tabular}{l|lll}
1 & 0.1060 .083
\end{tabular} \\
\hline \begin{tabular}{l|lll}
2 & 0.062 & 0.102
\end{tabular} & 20.0680 .097 & \begin{tabular}{l|ll|}
1 & 0.070 & 0.091
\end{tabular} & 10.0710 .087 & 20.0320 .088 & \begin{tabular}{l|lll}
2 & 0.028 & 0.085
\end{tabular} & \begin{tabular}{l|lll}
2 & 0.128 & 0.083
\end{tabular} \\
\hline $\begin{array}{lll}3 & 0.056 & 0.103\end{array}$ & 30.1090 .099 & 30.0390 .093 & \begin{tabular}{l|ll}
3 & 0.049 & 0.088
\end{tabular} & 30.0570 .090 & 30.0610 .087 & $\begin{array}{llll}30.033 & 0.086\end{array}$ \\
\hline a10 0.1360 .125 & 100.1000 .123 & 100.1230 .112 & 140.1020 .114 & 100.0900 .117 & 100.1040 .116 & 540.098 \\
\hline 90.1300 .131 & \begin{tabular}{l|lll}
9 & 0.095 & 0.129
\end{tabular} & \begin{tabular}{l|ll}
9 & 0.116 & 0.116
\end{tabular} & $\begin{array}{llll}9 & 0.114 & 0.117\end{array}$ & 90.1080 .123 & 90.1020 .122 & 100.1450 .116 \\
\hline 140.1270 .133 & 140.0920 .130 & \begin{tabular}{l|lll}
14 & 0.111 & 0.117
\end{tabular} & $\begin{array}{llllll}10 & 0.122 & 0.117\end{array}$ & $14 \quad 0.0980 .124$ & 140.0900 .124 & 140.1820 .118 \\
\hline $\begin{array}{llll}7 & 0.124 & 0.134\end{array}$ & \begin{tabular}{l|lll}
7 & 0.105 & 0.133
\end{tabular} & $7 \mid \begin{array}{ll}0.108 & 0.121\end{array}$ & $\begin{array}{llll}7 & 0.130 & 0.122\end{array}$ & 40.1370 .127 & \begin{tabular}{l|lll}
7 & 0.124 & 0.129
\end{tabular} & 90.2140 .120 \\
\hline \begin{tabular}{l|ll|l}
4 & 0.130 & 0.134
\end{tabular} & 40.1010 .133 & \begin{tabular}{l|ll|}
6 & 0.126 & 0.121
\end{tabular} & $11 \mid 0.1160 .124$ & 70.1140 .128 & \begin{tabular}{l|ll}
6 & 0.129 & 0.130
\end{tabular} & 70.2000 .125 \\
\hline \begin{tabular}{l|ll}
6 & 0.1390 .135
\end{tabular} & 60.1190 .134 & \begin{tabular}{l|ll}
4 & 0.116 & 0.122
\end{tabular} & \begin{tabular}{l|lll}
6 & 0.127 & 0.125
\end{tabular} & 60.1190 .128 & 40.1440 .131 & 60.1960 .127 \\
\hline \begin{tabular}{l|ll}
11 & 0.1180 .139
\end{tabular} & 110.1160 .137 & $\begin{array}{lll}11 & 0.102 & 0.124\end{array}$ & \begin{tabular}{l|lll}
13 & 0.101 & 0.129
\end{tabular} & 110.1190 .133 & 110.1200 .134 & \begin{tabular}{l|lll}
11 & 0.200 & 0.130
\end{tabular} \\
\hline $\begin{array}{llll}8 & 0.150 & 0.139\end{array}$ & 80.1440 .139 & 80.1370 .126 & \begin{tabular}{l|lll}
8 & 0.130 & 0.129
\end{tabular} & 80.1290 .135 & 80.1380 .137 & \begin{tabular}{l|l|l}
4 & 0.1180 .130
\end{tabular} \\
\hline $\begin{array}{lllllll}13 & 0.128 & 0.142\end{array}$ & \begin{tabular}{l|ll|}
12 & 0.129 & 0.142
\end{tabular} & \begin{tabular}{l|ll|}
13 & 0.111 & 0.128
\end{tabular} & \begin{tabular}{l|ll|l}
12 & 0.164 & 0.129
\end{tabular} & \begin{tabular}{l|ll|}
12 & 0.162 & 0.138
\end{tabular} & \begin{tabular}{l|ll|l}
12 & 0.160 & 0.139
\end{tabular} & 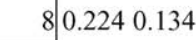 \\
\hline \begin{tabular}{l|lll}
12 & 0.132 & 0.142
\end{tabular} & $13 \mid 0.176 \quad 0.142$ & \begin{tabular}{l|ll}
12 & 0.116 & 0.128
\end{tabular} & \begin{tabular}{l|ll|}
4 & 0.128 & 0.129
\end{tabular} & 50.139 & 130.1350 .140 & \begin{tabular}{l|l}
12 & 0.1960 .135
\end{tabular} \\
\hline 150.1330 .146 & 150.1940 .147 & 300.1260 .152 & 150.1210 .133 & $\begin{array}{lllll}13 & 0.130 & 0.140\end{array}$ & 50.147 & $\begin{array}{llll}13 & 0.098 & 0.136\end{array}$ \\
\hline $\begin{array}{llll}30 & 0.148 & 0.172\end{array}$ & 50.154 & \begin{tabular}{l|lll}
27 & 0.191 & 0.162
\end{tabular} & 50.161 & 150.1750 .147 & 150.1780 .147 & 150.0940 .142 \\
\hline b5 0.172 & 300.1530 .174 & $5 \mid 0.163$ & 300.2040 .166 & 300.1740 .174 & 300.1800 .175 & 50.166 \\
\hline \begin{tabular}{l|lll}
27 & 0.203 & 0.180
\end{tabular} & 270.1930 .184 & \begin{tabular}{l|ll}
19 & 0.159 & 0.168
\end{tabular} & 360.1840 .177 & 270.1840 .184 & 270.1900 .188 & \begin{tabular}{ll|l}
30 & 0.164
\end{tabular} \\
\hline \begin{tabular}{l|l|l|l}
19 & 0.175 & 0.181
\end{tabular} & 190.1690 .187 & \begin{tabular}{l|ll}
18 & 0.1860 .169
\end{tabular} & 340.1950 .179 & 190.1960 .186 & \begin{tabular}{l|l|l|l|l|l|l|l|}
34 & 0.192 & 0.196
\end{tabular} & 270.2070 .185 \\
\hline 180.1990 .186 & $\begin{array}{llll}18 & 0.191 & 0.191\end{array}$ & $\begin{array}{lllllll}34 & 0.192 & 0.169\end{array}$ & \begin{tabular}{l|lll}
26 & 0.189 & 0.184
\end{tabular} & 180.2080 .192 & \begin{tabular}{l|ll}
19 & 0.205 & 0.196
\end{tabular} & 340.1960 .1 \\
\hline 340.20 & $\begin{array}{llll}34 & 0.193 & 0.192\end{array}$ & $26 \quad 0.1910 .172$ & \begin{tabular}{l|lll}
27 & 0.189 & 0.184
\end{tabular} & 360.196 & 180.2090 .196 & 190.0280 \\
\hline \begin{tabular}{l|ll|}
26 & 0.205 & 0.190
\end{tabular} & $26|0.1960 .195|$ & \begin{tabular}{l|lll}
29 & 0.181 & 0.173
\end{tabular} & 290.2040 .186 & \begin{tabular}{l|ll|}
26 & 0.196 & 0.198
\end{tabular} & 230.199 & \begin{tabular}{l|lll}
18 & 0.209 & 0.193
\end{tabular} \\
\hline $\begin{array}{llll}32 & 0.210 & 0.190\end{array}$ & \begin{tabular}{l|lll}
32 & 0.204 & 0.195
\end{tabular} & 320.1970 .175 & \begin{tabular}{ll|ll|}
18 & 0.189 & 0.188
\end{tabular} & $\begin{array}{llll}29 & 0.239 & 0.198\end{array}$ & \begin{tabular}{l|lll}
26 & 0.193 & 0.200
\end{tabular} & \begin{tabular}{l|lll}
29 & 0.221 & 0.193
\end{tabular} \\
\hline 290.1960 .193 & 290.1910 .197 & 360.1860 .178 & \begin{tabular}{l|lll|}
39 & 0.187 & 0.189
\end{tabular} & \begin{tabular}{l|l|ll}
32 & 0.197 & 0.199
\end{tabular} & 290.2300 .202 & \begin{tabular}{l|ll}
36 & 0.1950 .194
\end{tabular} \\
\hline \begin{tabular}{l|lll}
36 & 0.201 & 0.196
\end{tabular} & 360.1930 .199 & \begin{tabular}{l|ll}
25 & 0.204 & 0.182
\end{tabular} & 320.2050 .191 & 360.1790 .203 & \begin{tabular}{l|ll|}
32 & 0.220 & 0.205
\end{tabular} & \begin{tabular}{l|lll}
26 & 0.232 & 0.194
\end{tabular} \\
\hline \begin{tabular}{l|l|l|l}
17 & 0.209 & 0.196
\end{tabular} & 170.2090 .202 & \begin{tabular}{l|lll}
31 & 0.205 & 0.182
\end{tabular} & \begin{tabular}{l|l|l}
19 & 0.1940 .194
\end{tabular} & $\begin{array}{lll}17 & 0.213 & 0.205\end{array}$ & \begin{tabular}{l|lll}
36 & 0.181 & 0.206
\end{tabular} & 320.2420 .197 \\
\hline $\begin{array}{llll}25 & 0.217 & 0.198\end{array}$ & \begin{tabular}{l|lll}
25 & 0.223 & 0.204
\end{tabular} & 170.1970 .183 & \begin{tabular}{l|lll}
31 & 0.200 & 0.195
\end{tabular} & 250.2000 .208 & \begin{tabular}{l|lll}
25 & 0.212 & 0.213
\end{tabular} & \begin{tabular}{l|lll}
25 & 0.207 & 0.204
\end{tabular} \\
\hline $\begin{array}{lllll}31 & 0.218 & 0.199\end{array}$ & \begin{tabular}{l|ll}
31 & 0.209 & 0.205
\end{tabular} & 390.1980 .186 & \begin{tabular}{l|lll}
25 & 0.202 & 0.195
\end{tabular} & $\begin{array}{llll}31 & 0.228 & 0.212\end{array}$ & $\begin{array}{lllll}31 & 0.232 & 0.213\end{array}$ & \begin{tabular}{l|lll}
39 & 0.214 & 0.205
\end{tabular} \\
\hline 390.2120 .203 & $\begin{array}{lll}39 & 0.208 & 0.208\end{array}$ & \begin{tabular}{l|l|l|l}
33 & 0.1870 .193
\end{tabular} & 170.1980 .199 & $\begin{array}{lll}39 & 0.213 & 0.215\end{array}$ & $\begin{array}{llll}17 & 0.222 & 0.214\end{array}$ & \begin{tabular}{l|lll}
17 & 0.203 & 0.206
\end{tabular} \\
\hline \begin{tabular}{l|lll}
33 & 0.202 & 0.207
\end{tabular} & \begin{tabular}{l|ll|}
33 & 0.206 & 0.214
\end{tabular} & $\begin{array}{lll}38 & 0.200 & 0.193\end{array}$ & 380.2470 .204 & 230.217 & $\begin{array}{lll}39 & 0.221 & 0.217\end{array}$ & \begin{tabular}{l|lll}
31 & 0.263 & 0.206
\end{tabular} \\
\hline \begin{tabular}{l|lll}
28 & 0.217 & 0.207
\end{tabular} & \begin{tabular}{l|ll|}
28 & 0.219 & 0.214
\end{tabular} & \begin{tabular}{l|lll}
16 & 0.228 & 0.193
\end{tabular} & 280.2240 .206 & \begin{tabular}{l|ll}
16 & 0.243 & 0.218
\end{tabular} & 240.220 & 240. \\
\hline $\begin{array}{llll}16 & 0.236 & 0.207\end{array}$ & \begin{tabular}{l|lll}
16 & 0.234 & 0.214
\end{tabular} & 150.1160 .193 & $\begin{array}{llll}35 & 0.222 & 0.209\end{array}$ & \begin{tabular}{ll|ll|}
33 & 0.256 & 0.218
\end{tabular} & \begin{tabular}{l|lll}
38 & 0.247 & 0.226
\end{tabular} & 230.210 \\
\hline 380.2140 .212 & \begin{tabular}{ll|lll}
38 & 0.213 & 0.217
\end{tabular} & 280.2030 .194 & \begin{tabular}{l|ll|l}
16 & 0.235 & 0.209
\end{tabular} & \begin{tabular}{ll|ll|}
38 & 0.259 & 0.220
\end{tabular} & \begin{tabular}{l|lll}
16 & 0.250 & 0.226
\end{tabular} & 380.2240 .214 \\
\hline 210.214 & 210.217 & $\begin{array}{lll}21 & 0.200\end{array}$ & 200.2460 .209 & $\begin{array}{ll}24 & 0.221\end{array}$ & \begin{tabular}{llll|}
33 & 0.263 & 0.226
\end{tabular} & \begin{tabular}{l|lll}
28 & 0.264 & 0.216
\end{tabular} \\
\hline \begin{tabular}{l|lll}
20 & 0.247 & 0.214
\end{tabular} & 350.2380 .221 & \begin{tabular}{l|lll}
35 & 0.211 & 0.201
\end{tabular} & \begin{tabular}{l|lll}
33 & 0.214 & 0.210
\end{tabular} & $280.233 \quad 0.223$ & \begin{tabular}{l|lll}
28 & 0.240 & 0.227
\end{tabular} & \begin{tabular}{l|ll}
16 & 0.199 & 0.217
\end{tabular} \\
\hline 350.2240 .215 & 200.2510 .221 & 200.2400 .201 & \begin{tabular}{l|ll|}
22 & 0.243 & 0.221
\end{tabular} & $\begin{array}{llll}35 & 0.247 & 0.228\end{array}$ & \begin{tabular}{l|lll}
35 & 0.248 & 0.235
\end{tabular} & \begin{tabular}{l|lll}
33 & 0.254 & 0.218
\end{tabular} \\
\hline
\end{tabular}


Table 3 (Continued)

\begin{tabular}{|c|c|c|c|c|c|c|}
\hline $22 \mid 0.2590 .223$ & \begin{tabular}{l|l|}
23 & 0.225
\end{tabular} & \begin{tabular}{l|ll|}
22 & 0.252 & 0.211
\end{tabular} & 370.2460 .221 & \begin{tabular}{l|ll}
20 & 0.230 & 0.228
\end{tabular} & \begin{tabular}{l|lll}
20 & 0.233 & 0.235
\end{tabular} & \begin{tabular}{l|lll}
20 & 0.222 & 0.223
\end{tabular} \\
\hline $\begin{array}{llll}37 & 0.243 & 0.223\end{array}$ & \begin{tabular}{l|l|}
24 & 0.225
\end{tabular} & $37 \begin{array}{lll}0.233 & 0.211\end{array}$ & \begin{tabular}{l|l|}
23 & 0.225
\end{tabular} & \begin{tabular}{l|lll}
22 & 0.245 & 0.242
\end{tabular} & \begin{tabular}{l|ll|}
22 & 0.253 & 0.247
\end{tabular} & \begin{tabular}{l|lll}
35 & 0.233 & 0.224
\end{tabular} \\
\hline 240.235 & $22 \mid \begin{array}{lll}22 & 0.279 & 0.231\end{array}$ & 540.226 & 240.226 & 370.2800 .242 & $37 \mid \begin{array}{lll}0.281 & 0.247\end{array}$ & \begin{tabular}{l|lll}
22 & 0.228 & 0.235
\end{tabular} \\
\hline \begin{tabular}{l|l|l|}
54 & 0.241
\end{tabular} & $\begin{array}{llll}37 & 0.265 & 0.232\end{array}$ & \begin{tabular}{l|l|}
24 & 0.227 \\
\end{tabular} & 800.2890 .255 & \begin{tabular}{l|l}
21 & 0.267
\end{tabular} & 210.266 & 370.2400 .236 \\
\hline 230.242 & 540.250 & $\begin{array}{llll}53 & 0.250 & 0.232\end{array}$ & 210.259 & \begin{tabular}{l|l|l|l}
53 & 0.289 & 0.268
\end{tabular} & \begin{tabular}{l|lll}
53 & 0.308 & 0.272
\end{tabular} & 210.240 \\
\hline $\begin{array}{llll}53 & 0.263 & 0.253\end{array}$ & \begin{tabular}{l|lll|}
53 & 0.266 & 0.264
\end{tabular} & $\begin{array}{lllll}73 & 0.273 & 0.234\end{array}$ & \begin{tabular}{l|ll|}
72 & 0.282 & 0.261
\end{tabular} & \begin{tabular}{l|lll}
73 & 0.260 & 0.269
\end{tabular} & \begin{tabular}{l|lll}
73 & 0.258 & 0.273
\end{tabular} & 450.254 \\
\hline \begin{tabular}{ll|ll|}
73 & 0.284 & 0.257
\end{tabular} & $\begin{array}{llll}73 & 0.288 & 0.266\end{array}$ & 230.235 & \begin{tabular}{l|lll}
73 & 0.302 & 0.262
\end{tabular} & 690.2610 .274 & 690.2540 .278 & 610.256 \\
\hline \begin{tabular}{l|lll}
69 & 0.262 & 0.258
\end{tabular} & 690.2810 .268 & 690.2470 .238 & 690.3010 .265 & $\begin{array}{lll}50 & 0.274 & 0.278\end{array}$ & $\begin{array}{lll}50 & 0.283 & 0.287\end{array}$ & 730.2160 .264 \\
\hline 500.2790 .261 & 500.2880 .272 & $\begin{array}{llll}50 & 0.268 & 0.247\end{array}$ & \begin{tabular}{l|l}
54 & 0.274
\end{tabular} & \begin{tabular}{l|l|l}
72 & 0.287 & 0.289
\end{tabular} & 720.2830 .291 & \begin{tabular}{l|lll}
69 & 0.227 & 0.264
\end{tabular} \\
\hline 720.2930 .274 & 720.2970 .281 & $\begin{array}{llll}72 & 0.282 & 0.253\end{array}$ & \begin{tabular}{l|ll|}
53 & 0.277 & 0.275 \\
\end{tabular} & \begin{tabular}{l|l|l}
54 & 0.290
\end{tabular} & $\begin{array}{ll}57 & 0.296\end{array}$ & \begin{tabular}{l|l|}
52 & 0.268
\end{tabular} \\
\hline \begin{tabular}{l|lll}
62 & 0.331 & 0.277
\end{tabular} & 520.284 & 620.3270 .256 & \begin{tabular}{l|lll}
50 & 0.297 & 0.278
\end{tabular} & \begin{tabular}{l|lll}
51 & 0.290 & 0.296
\end{tabular} & \begin{tabular}{l|lll|}
62 & 0.329 & 0.297
\end{tabular} & \begin{tabular}{l|lll}
53 & 0.255 & 0.269
\end{tabular} \\
\hline \begin{tabular}{l|lll}
51 & 0.271 & 0.278
\end{tabular} & \begin{tabular}{l|lll}
62 & 0.332 & 0.287
\end{tabular} & $\begin{array}{lll}80 & 0.273 & 0.267\end{array}$ & \begin{tabular}{l|lll}
68 & 0.302 & 0.280
\end{tabular} & $\begin{array}{llll}75 & 0.293 & 0.296\end{array}$ & 800.2870 .304 & \begin{tabular}{l|lll}
50 & 0.229 & 0.272
\end{tabular} \\
\hline \begin{tabular}{l|lll}
75 & 0.289 & 0.278
\end{tabular} & \begin{tabular}{l|lll}
51 & 0.278 & 0.289
\end{tabular} & \begin{tabular}{l|ll|}
49 & 0.307 & 0.269
\end{tabular} & \begin{tabular}{l|lll}
49 & 0.302 & 0.290
\end{tabular} & \begin{tabular}{ll|lll}
62 & 0.340 & 0.296
\end{tabular} & \begin{tabular}{l|lll}
68 & 0.292 & 0.308
\end{tabular} & $\begin{array}{ll}57 & 0.273\end{array}$ \\
\hline $\begin{array}{lllll}49 & 0.314 & 0.283\end{array}$ & $\begin{array}{lllll}80 & 0.291 & 0.289\end{array}$ & \begin{tabular}{l|lll|}
68 & 0.284 & 0.270
\end{tabular} & \begin{tabular}{l|ll}
62 & 0.327 & 0.291
\end{tabular} & 800.2850 .299 & $\begin{array}{lllll}49 & 0.324 & 0.309\end{array}$ & $\begin{array}{lll}43 & 0.274\end{array}$ \\
\hline 520.283 & $\begin{array}{llll}75 & 0.307 & 0.290 \\
\end{array}$ & \begin{tabular}{l|lll}
51 & 0.258 & 0.271
\end{tabular} & 670.2980 .294 & 570.302 & $\begin{array}{lll}59 & 0.310\end{array}$ & $\begin{array}{lll}59 & 0.276\end{array}$ \\
\hline \begin{tabular}{l|lll}
80 & 0.286 & 0.284
\end{tabular} & \begin{tabular}{l|lll}
68 & 0.306 & 0.294
\end{tabular} & \begin{tabular}{l|ll|}
75 & 0.277 & 0.271
\end{tabular} & \begin{tabular}{l|lll}
51 & 0.268 & 0.296
\end{tabular} & \begin{tabular}{ll|lll}
48 & 0.335 & 0.304
\end{tabular} & $75 \mid \begin{array}{llll} & 0.304 & 0.311\end{array}$ & \begin{tabular}{l|lll}
72 & 0.223 & 0.277
\end{tabular} \\
\hline $\begin{array}{llll}68 & 0.296 & 0.285\end{array}$ & $\begin{array}{llll}49 & 0.314 & 0.294\end{array}$ & \begin{tabular}{l|l|}
52 & 0.271
\end{tabular} & \begin{tabular}{l|lll}
75 & 0.309 & 0.296
\end{tabular} & \begin{tabular}{l|lll}
68 & 0.291 & 0.305
\end{tabular} & \begin{tabular}{l|lll|}
51 & 0.325 & 0.311
\end{tabular} & 800.1500 .281 \\
\hline $\begin{array}{lllll}71 & 0.280 & 0.287\end{array}$ & $\begin{array}{lllll}48 & 0.301 & 0.298\end{array}$ & \begin{tabular}{llll|l|l|l|}
48 & 0.280 & 0.272
\end{tabular} & \begin{tabular}{l|l|l}
52 & 0.296
\end{tabular} & \begin{tabular}{l|lll}
49 & 0.318 & 0.305
\end{tabular} & \begin{tabular}{lllll|lll}
48 & 0.332 & 0.313
\end{tabular} & \begin{tabular}{l|ll}
62 & 0.256 & 0.283
\end{tabular} \\
\hline \begin{tabular}{l|l|l|l|l|l|l|}
46 & 0.282 & 0.287
\end{tabular} & \begin{tabular}{l|ll|l|l|l|}
71 & 0.291 & 0.299
\end{tabular} & \begin{tabular}{llll|l|l|l|}
71 & 0.268 & 0.275
\end{tabular} & \begin{tabular}{l|l|l|l|l|}
48 & 0.297 & 0.298 \\
\end{tabular} & \begin{tabular}{ll|ll}
46 & 0.328 & 0.306
\end{tabular} & \begin{tabular}{l|ll|l}
46 & 0.337 & 0.315
\end{tabular} & \begin{tabular}{l|lll}
68 & 0.231 & 0.290 \\
\end{tabular} \\
\hline \begin{tabular}{l|lll|}
48 & 0.291 & 0.287
\end{tabular} & \begin{tabular}{l|lll}
46 & 0.291 & 0.299
\end{tabular} & $460.2^{\prime}$ & $\begin{array}{llll}79 & 0.321 & 0.302\end{array}$ & 710.3290 .306 & \begin{tabular}{l|ll}
71 & 0.348 & 0.315
\end{tabular} & 650.293 \\
\hline 670.3000 .294 & 670.3120 .303 & $\begin{array}{lll}67 & 0.289 & 0.279\end{array}$ & \begin{tabular}{ll|ll|}
58 & 0.328 & 0.302 \\
\end{tabular} & 590.310 & \begin{tabular}{l|l|l}
54 & 0.315
\end{tabular} & 510.2030 .296 \\
\hline 470.3250 .301 & 470.3320 .313 & 470.3180 .297 & 710.2690 .306 & $\begin{array}{lll}67 & 0.322 & 0.312\end{array}$ & $\begin{array}{lll}67 & 0.322 & 0.318\end{array}$ & 750.2530 .296 \\
\hline \begin{tabular}{l|ll|}
74 & 0.318 & 0.309
\end{tabular} & \begin{tabular}{l|lll}
74 & 0.328 & 0.319
\end{tabular} & \begin{tabular}{l|lll|l|l|l|l|}
74 & 0.309 & 0.299 \\
\end{tabular} & \begin{tabular}{l|lll}
46 & 0.288 & 0.306
\end{tabular} & \begin{tabular}{l|l}
43 & 0.315
\end{tabular} & 650.322 & \begin{tabular}{l|lll}
49 & 0.280 & 0.296
\end{tabular} \\
\hline $76 \mid \begin{array}{lll}0.306 & 0.311\end{array}$ & \begin{tabular}{l|l|l}
45 & 0.319
\end{tabular} & \begin{tabular}{ll|l|}
76 & 0.296 & 0.300 \\
\end{tabular} & $\begin{array}{lll}70 & 0.307 \\
\end{array}$ & \begin{tabular}{l|l}
52 & 0.3 \\
\end{tabular} & 430. & \begin{tabular}{l|lll}
48 & 0.288 & 0.298
\end{tabular} \\
\hline \begin{tabular}{l|l|l|l|l|}
79 & 0.327 & 0.312
\end{tabular} & 440.319 & \begin{tabular}{ll|ll|}
79 & 0.320 & 0.304
\end{tabular} & $\begin{array}{lll}65 & 0.308\end{array}$ & 450.323 & \begin{tabular}{l|l|}
63 & 0.328
\end{tabular} & $\begin{array}{llll}67 & 0.283 & 0.301\end{array}$ \\
\hline 580.3380 .312 & 790.3340 .321 & \begin{tabular}{l|lll}
58 & 0.333 & 0.304
\end{tabular} & 470.3150 .308 & 470.3220 .326 & $\begin{array}{lll}61 & 0.329\end{array}$ & \\
\hline $66 \mid \begin{array}{lll}6 & 0.335 & 0.316\end{array}$ & \begin{tabular}{l|l|l|}
58 & 0.340 & 0.321 \\
\end{tabular} & $\begin{array}{lll}70 & 0.311 \\
\end{array}$ & \begin{tabular}{|l|l|}
44 & 0.310 \\
\end{tabular} & 760.3570 .329 & \begin{tabular}{|l|l|}
52 & 0.330 \\
\end{tabular} & \begin{tabular}{l|lll}
71 & 0.270 & 0.303
\end{tabular} \\
\hline \begin{tabular}{ll|ll|}
42 & 0.334 & 0.317 \\
\cline { 1 - 2 }
\end{tabular} & $\begin{array}{ll}430.322\end{array}$ & \begin{tabular}{l|l}
44 & 0.312
\end{tabular} & \begin{tabular}{l|l|l}
45 & 0.311
\end{tabular} & \begin{tabular}{l|l|l|l}
74 & 0.356 & 0.334
\end{tabular} & \begin{tabular}{l|l|l}
45 & 0.335
\end{tabular} & \begin{tabular}{ll|l}
46 & 0.2460 .304
\end{tabular} \\
\hline \begin{tabular}{l|l|l|}
444 & 0.319
\end{tabular} & 760.3170 .322 & \begin{tabular}{l|lll}
66 & 0.328 & 0.313
\end{tabular} & $\begin{array}{lll}59 & 0.311 \\
\end{array}$ & 440.334 & 470.3400 .335 & $\begin{array}{lll}47 & 0.275 & 0.315\end{array}$ \\
\hline $\begin{array}{lll}70 & 0.319\end{array}$ & 700.323 & $\begin{array}{lllll}42 & 0.329 & 0.313 \\
\end{array}$ & $780.340 \quad 0.322$ & 790.3390 .335 & $\begin{array}{lll}74 & 0.351 & 0.338\end{array}$ & $\begin{array}{lll}70 & 0.317 \\
\end{array}$ \\
\hline \begin{tabular}{l|l}
45 & 0.321
\end{tabular} & 650.326 & \begin{tabular}{|l|l|}
41 & 0.314 \\
\end{tabular} & \begin{tabular}{ll|ll|}
7 & 0.329 & 0.322 \\
\end{tabular} & 630.335 & \begin{tabular}{ll|ll|}
76 & 0.350 & 0.339 \\
\end{tabular} & \begin{tabular}{l|l|}
41 & 0.317
\end{tabular} \\
\hline $\begin{array}{lll}41 & 0.321 \\
\end{array}$ & \begin{tabular}{l|ll|}
66 & 0.346 & 0.328 \\
\end{tabular} & $\begin{array}{lll}45 & 0.316\end{array}$ & $\begin{array}{llll}66 & 0.322 & 0.326\end{array}$ & 580.3030 .335 & \begin{tabular}{l|lll|}
79 & 0.348 & 0.341
\end{tabular} & \begin{tabular}{l|l|}
44 & 0.317
\end{tabular} \\
\hline 430.324 & \begin{tabular}{l|ll|l}
42 & 0.348 & 0.329 \\
\end{tabular} & \begin{tabular}{ll|ll|}
78 & 0.319 & 0.318
\end{tabular} & \begin{tabular}{ll|ll|}
42 & 0.317 & 0.327 \\
\end{tabular} & $\begin{array}{ll}65 & 0.337 \\
\end{array}$ & \begin{tabular}{ll|ll|}
58 & 0.302 & 0.341 \\
\end{tabular} & $\begin{array}{lll}74 & 0.3170 .318 \\
\end{array}$ \\
\hline 780.3270 .325 & \begin{tabular}{l|l}
61 & 0.332
\end{tabular} & 430.318 & $\begin{array}{ll}61 & 0.327 \\
\end{array}$ & $\begin{array}{ll}61 & 0.340 \\
\end{array}$ & \begin{tabular}{l|lll}
42 & 0.345 & 0.350
\end{tabular} & 640.318 \\
\hline $\begin{array}{lll}55 & 0.333 & 0.328\end{array}$ & \begin{tabular}{l|l|l|}
41 & 0.333
\end{tabular} & $\begin{array}{llll}55 & 0.327 & 0.326\end{array}$ & 630.329 & 640.341 & $\begin{array}{llll}66 & 0.372 & 0.350\end{array}$ & \begin{tabular}{l|ll}
58 & 0.2710 .319
\end{tabular} \\
\hline \begin{tabular}{l|l|l}
65 & 0.331
\end{tabular} & \begin{tabular}{l|lll|}
78 & 0.340 & 0.334
\end{tabular} & 610.326 & 740.3090 .330 & \begin{tabular}{l|ll}
42 & 0.335 & 0.343
\end{tabular} & $\begin{array}{llll}78 & 0.331 & 0.354\end{array}$ & $\begin{array}{llll}79 & 0.268 & 0.320\end{array}$ \\
\hline \begin{tabular}{l|l|}
61 & 0.331
\end{tabular} & $\begin{array}{lll}59 & 0.334 \\
\end{array}$ & 650.327 & 430. & \begin{tabular}{l|lll}
66 & 0.361 & 0.343 \\
\end{tabular} & 640.354 & \begin{tabular}{l|lll}
76 & 0.312 & 0.322 \\
\end{tabular} \\
\hline $\begin{array}{lll}59 & 0.333 \\
\end{array}$ & 570.337 & $\begin{array}{lll}59 & 0.329 \\
\end{array}$ & \begin{tabular}{l|l|}
57 & 0.337
\end{tabular} & $\begin{array}{lll}78 & 0.343 & 0.346\end{array}$ & $\begin{array}{lll}70 & 0.357\end{array}$ & 960.325 \\
\hline 570.336 & 550.3510 .341 & 570.332 & \begin{tabular}{l|l|l|}
56 & 0.338 \\
\end{tabular} & \begin{tabular}{l|l|l|l}
55 & 0.354 & 0.356
\end{tabular} & \begin{tabular}{l|l|l}
44 & 0.359 \\
\end{tabular} & \begin{tabular}{l|l|l|}
56 & 0.327
\end{tabular} \\
\hline
\end{tabular}


Table 3 (Continued)

\begin{tabular}{|c|c|c|c|c|c|c|c|c|c|c|c|}
\hline $\begin{array}{llll}81 & 0.350 & 0.339\end{array}$ & 640.349 & 81 & 0.3450 .335 & & 0.342 & & 0.357 & & 0.3490 .363 & 111 & 0.331 \\
\hline 640.342 & \begin{tabular}{l|lll}
81 & 0.363 & 0.350
\end{tabular} & 103 & 0.3900 .336 & 41 & 0.342 & 81 & $\begin{array}{lll}0.377 & 0.365\end{array}$ & & 0.370 & 42 & 0.2870 .332 \\
\hline $\begin{array}{llll}77 & 0.341 & 0.343\end{array}$ & 630.353 & 64 & 0.338 & 81 & $\begin{array}{lll}0.355 & 0.344\end{array}$ & 41 & 0.367 & 81 & 0.3670 .370 & 66 & 0.3070 .332 \\
\hline $\begin{array}{lllll}40 & 0.325 & 0.344\end{array}$ & $\begin{array}{lllll}40 & 0.340 & 0.355\end{array}$ & 60 & 0.338 & 55 & $\begin{array}{lll}0.348 & 0.349\end{array}$ & 40 & 0.3730 .372 & 103 & 0.3720 .373 & 78 & 0.3000 .333 \\
\hline 600.344 & $\begin{array}{llll}77 & 0.360 & 0.355\end{array}$ & 121 & 0.3860 .339 & 77 & $\mid \begin{array}{lll}0.349 & 0.350\end{array}$ & 77 & 0.4090 .372 & 121 & 0.3640 .375 & 55 & 0.3080 .347 \\
\hline $\begin{array}{ll}56 & 0.346\end{array}$ & 600.358 & 56 & 0.341 & 40 & $\begin{array}{lll}0.335 & 0.351\end{array}$ & 104 & 0.3630 .373 & & 0.377 & 81 & 0.3190 .349 \\
\hline 630.352 & 560.363 & 104 & 0.3480 .344 & 121 & $\begin{array}{lll}0.419 & 0.362\end{array}$ & & 0.373 & 40 & 0.3850 .380 & 121 & 0.2920 .356 \\
\hline $\begin{array}{lllll}104 & 0.358 & 0.357\end{array}$ & $\begin{array}{lllll} & 0.376 & 0.369\end{array}$ & 40 & 0.3200 .347 & 60 & 0.364 & 103 & 0.3830 .374 & 77 & 0.4120 .380 & 60 & 0.358 \\
\hline \begin{tabular}{l|ll|}
103 & 0.396 & 0.358
\end{tabular} & \begin{tabular}{ll|lll}
103 & 0.407 & 0.369
\end{tabular} & 77 & 0.3340 .347 & 103 & 0.4130 .372 & 121 & 0.3740 .377 & 104 & $\begin{array}{lll}0.378 & 0.382\end{array}$ & 104 & 0.273 \\
\hline 1210.3930 .363 & 1210.4090 .371 & 63 & 0.348 & 104 & 0.3900 .383 & & 0.386 & & 0.391 & 77 & 0.3290 .359 \\
\hline 1020.3740 .385 & 1020.3930 .396 & 102 & 0.3660 .370 & 120 & 0.4090 .394 & 102 & 0.4180 .396 & 94 & 0.397 & 113 & 0.359 \\
\hline 1000.4130 .395 & 1000.4270 .405 & 101 & 0.4120 .389 & 101 & $\begin{array}{lll}0.408 & 0.403\end{array}$ & 94 & 0.400 & 102 & $\begin{array}{lll}0.423 & 0.404\end{array}$ & 40 & 0.3070 .360 \\
\hline 1010.4160 .401 & 1010.4240 .410 & 109 & 0.4330 .395 & 100 & $\begin{array}{lll}0.414 & 0.403\end{array}$ & 111 & 0.412 & 111 & 0.405 & 103 & 0.3380 .360 \\
\hline 1190.4170 .405 & 1200.4320 .414 & 100 & 0.4090 .396 & 102 & 0.4140 .408 & 100 & 0.3850 .413 & 113 & 0.408 & 94 & 0.373 \\
\hline 980.3980 .405 & 1190.4350 .414 & 120 & 0.4160 .398 & 98 & $\begin{array}{lll}0.394 & 0.414\end{array}$ & 96 & 0.413 & 112 & 0.409 & 92 & 0.376 \\
\hline 1090.4340 .406 & \begin{tabular}{l|ll|}
98 & 0.416 & 0.414
\end{tabular} & 119 & 0.4120 .400 & 119 & 0.4250 .416 & 113 & 0.414 & 92 & 0.417 & 90 & 0.385 \\
\hline 1200.4200 .408 & $\begin{array}{llll}109 & 0.446 & 0.417\end{array}$ & 98 & 0.3920 .400 & 93 & 0.418 & 101 & 0.4390 .418 & 101 & 0.4360 .418 & 102 & \\
\hline \begin{tabular}{ll|ll|}
88 & 0.428 & 0.411
\end{tabular} & 960.419 & 125 & 0.4040 .403 & 92 & 0.419 & 92 & 0.421 & 100 & 0.3930 .424 & 100 & 0.3410 .394 \\
\hline 960.411 & \begin{tabular}{l|lll|}
88 & 0.437 & 0.423
\end{tabular} & 91 & 0.408 & 96 & 0.419 & 112 & 0.422 & 120 & 0.4330 .424 & 91 & 0.397 \\
\hline 910.412 & \begin{tabular}{llll|}
125 & 0.422 & 0.424
\end{tabular} & 88 & 0.4280 .409 & 95 & 0.422 & 119 & $\begin{array}{lll}0.429 & 0.423\end{array}$ & 96 & 0.424 & 95 & 0.399 \\
\hline $\begin{array}{llll}125 & 0.409 & 0.414\end{array}$ & 890.426 & 96 & 0.409 & 109 & $\begin{array}{lll}0.456 & 0.427\end{array}$ & 120 & 0.4300 .423 & 109 & 0.4140 .425 & 120 & 0.3460 .400 \\
\hline 890.414 & 910.429 & 89 & 0.411 & 99 & $\begin{array}{lll}0.421 & 0.430\end{array}$ & 98 & 0.4020 .423 & 119 & $\begin{array}{lll}0.432 & 0.428\end{array}$ & 101 & 0.3950 .400 \\
\hline \begin{tabular}{l|l|}
82 & 0.423
\end{tabular} & 940.430 & 82 & 0.420 & 91 & 0.430 & 90 & 0.424 & 98 & 0.3990 .428 & 119 & 0.3760 .403 \\
\hline \begin{tabular}{l|l}
122 & 0.424
\end{tabular} & \begin{tabular}{l|lll|}
99 & 0.432 & 0.433
\end{tabular} & 122 & 0.421 & 127 & 0.4650 .430 & 125 & $\begin{array}{lll}0.441 & 0.426\end{array}$ & 125 & 0.4440 .431 & 98 & 0.3590 .403 \\
\hline \begin{tabular}{l|lll}
99 & 0.419 & 0.425
\end{tabular} & \begin{tabular}{l|l|}
93 & 0.433
\end{tabular} & 99 & 0.4140 .424 & 90 & 0.433 & 109 & 0.4250 .427 & 91 & 0.433 & 109 & 0.4030 .407 \\
\hline \begin{tabular}{l|l|}
84 & 0.427
\end{tabular} & \begin{tabular}{l|l|}
84 & 0.437 \\
\end{tabular} & 116 & 0.4500 .424 & 124 & 0.4470 .435 & 88 & 0.4380 .431 & 95 & 0.434 & 112 & 0.410 \\
\hline 940.427 & \begin{tabular}{l|l|}
95 & 0.438
\end{tabular} & 84 & 0.427 & 94 & 0.435 & 95 & 0.434 & 88 & 0.4390 .438 & 125 & \\
\hline 930.431 & $115|0.4700 .442|$ & 94 & 0.428 & 125 & $\begin{array}{lll}0.440 & 0.436\end{array}$ & 93 & 0.435 & 90 & 0.438 & 88 & 0.3930 .416 \\
\hline 950.433 & $124 \mid 0.4590 .443$ & 124 & $\begin{array}{lll}0.453 & 0.432\end{array}$ & 122 & 0.437 & 91 & & 93 & & 93 & \\
\hline \begin{tabular}{ll|lll}
116 & 0.447 & 0.434
\end{tabular} & $\begin{array}{ll}92 & 0.443\end{array}$ & 93 & 0.433 & 84 & 0.440 & 83 & 0.441 & 83 & & 83 & 0.419 \\
\hline $115 \mid \begin{array}{lll}0.460 & 0.434\end{array}$ & \begin{tabular}{l|l|}
122 & 0.443
\end{tabular} & 95 & 0.434 & 83 & 0.441 & 99 & 0.4410 .443 & 99 & $\begin{array}{lll}0.451 & 0.447\end{array}$ & 107 & 0.420 \\
\hline $124 \mid 0.452 \quad 0.436$ & $\begin{array}{lll}83 & 0.443\end{array}$ & 115 & 0.4620 .435 & 112 & 0.443 & 124 & 0.4620 .453 & 116 & 0.4760 .452 & 99 & $\begin{array}{lll}0.400 & 0.422\end{array}$ \\
\hline 830.436 & \begin{tabular}{ll|ll|}
116 & 0.449 & 0.444
\end{tabular} & 83 & 0.436 & 113 & 0.443 & 84 & 0.453 & 124 & $\begin{array}{lll}0.455 & 0.454\end{array}$ & 116 & 0.4540 .430 \\
\hline 1130.438 & \begin{tabular}{ll|l}
82 & 0.444
\end{tabular} & 113 & 0.438 & 89 & 0.443 & 116 & 0.4880 .454 & 84 & 0.455 & 115 & 0.4100 .430 \\
\hline $\begin{array}{ll}92 & 0.439\end{array}$ & $\begin{array}{lll}113 & 0.444\end{array}$ & 92 & 0.440 & 88 & $\begin{array}{lll}0.424 & 0.444\end{array}$ & 89 & 0.455 & 117 & 0.456 & 124 & 0.4110 .432 \\
\hline \begin{tabular}{l|l}
112 & 0.445
\end{tabular} & 1120.448 & 111 & 0.446 & 115 & 0.4460 .445 & 117 & 0.455 & 115 & 0.4620 .458 & 84 & 0.432 \\
\hline 1110.445 & $\begin{array}{ll}111 & 0.451\end{array}$ & 90 & 0.446 & 117 & 0.447 & 115 & 0.4570 .457 & 107 & 0.461 & 89 & 0.438 \\
\hline 900.446 & 900.452 & 112 & 0.446 & 111 & 0.448 & 127 & 0.4520 .465 & 89 & 0.463 & 127 & 0.3590 .439 \\
\hline $127 \mid 0.4380 .449$ & 1270.4530 .454 & 110 & 0.451 & 82 & 0.448 & 86 & 0.4780 .466 & 127 & 0.4430 .469 & 117 & 0.441 \\
\hline
\end{tabular}


Table 3 (Continued)

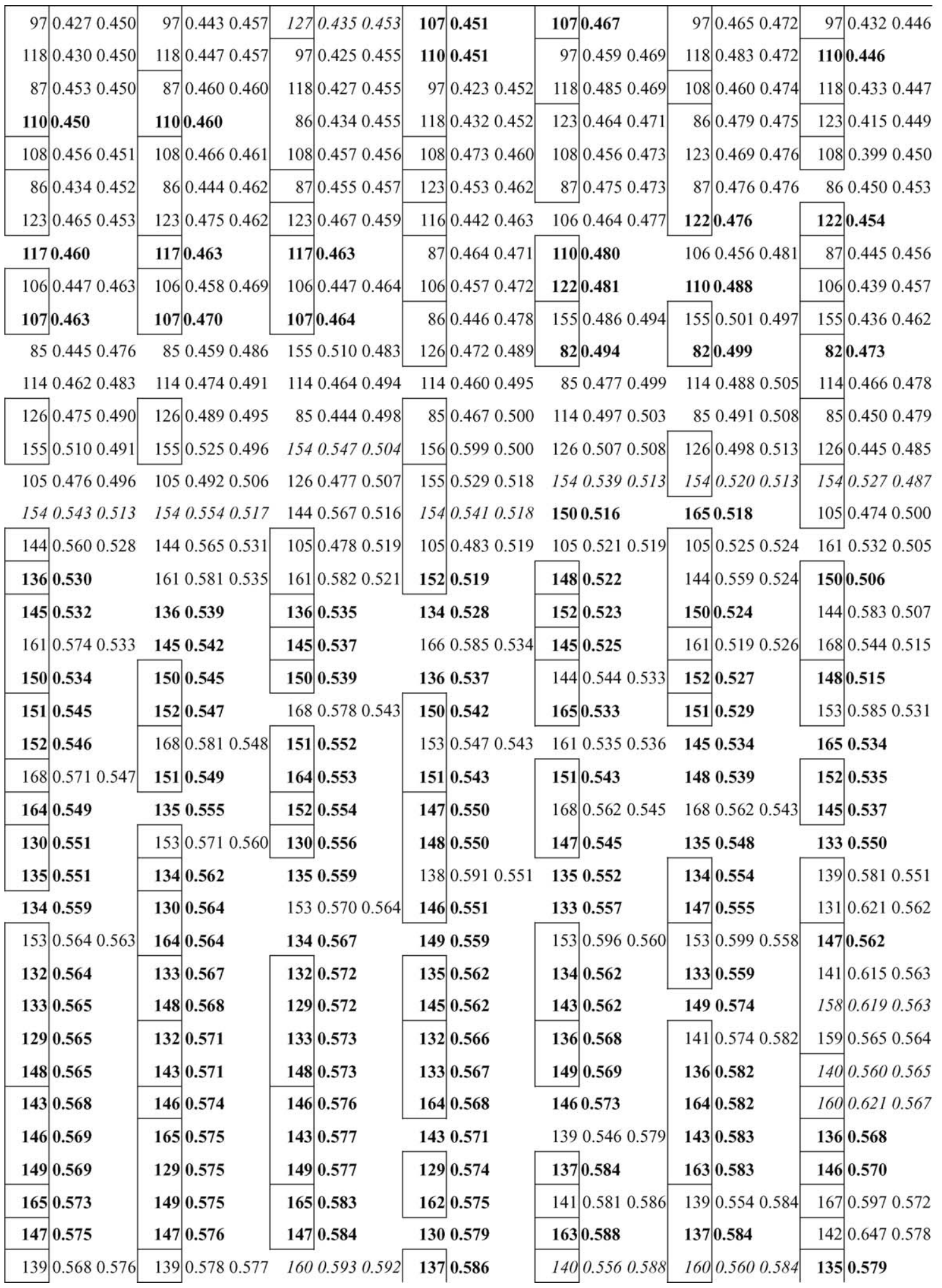


Table 3 (Continued)

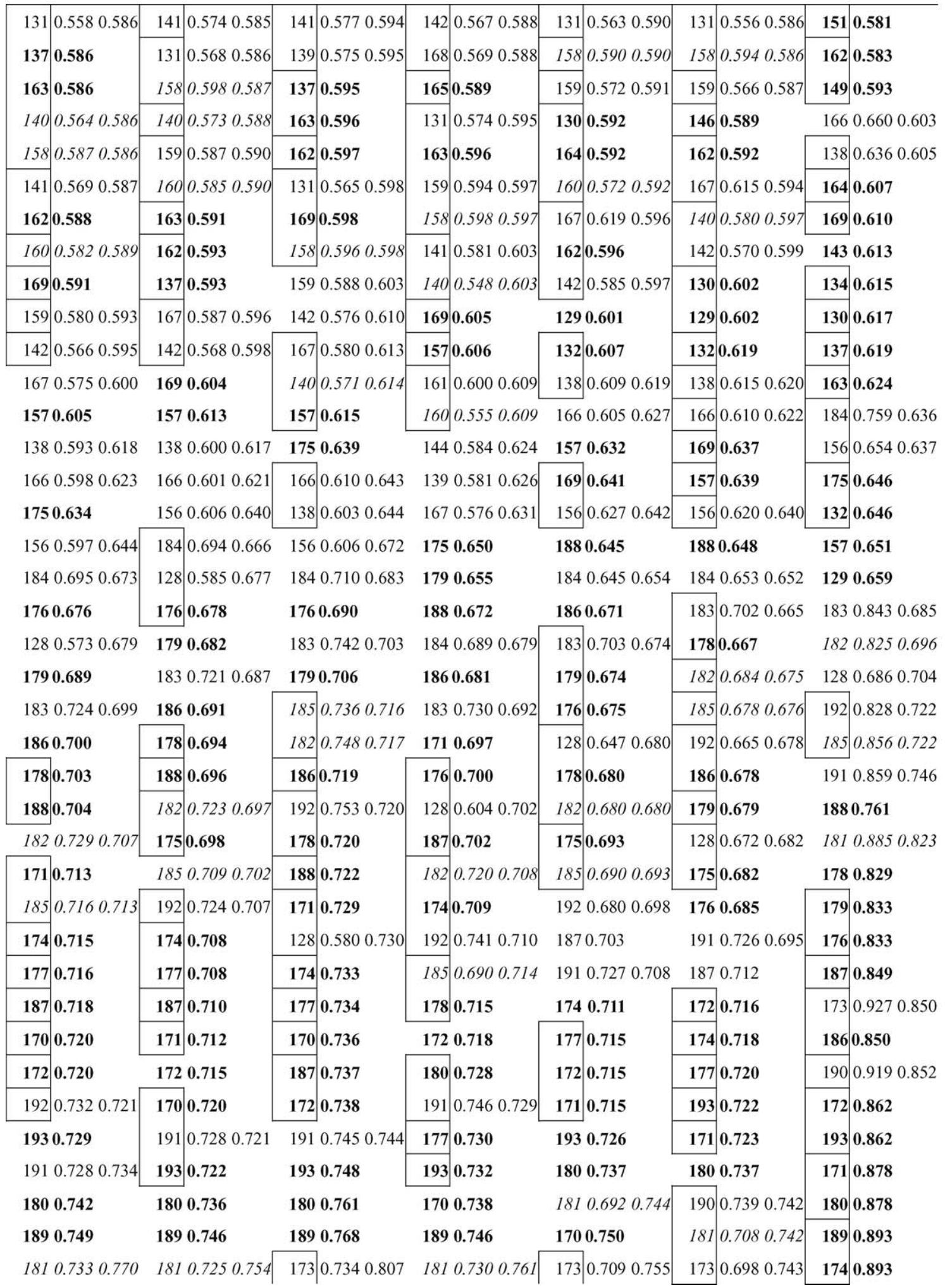


Table 3 (Continued)

\begin{tabular}{|c|c|c|c|c|c|c|}
\hline $\begin{array}{llll}173 & 0.715 & 0.779\end{array}$ & \begin{tabular}{l|lll|}
173 & 0.708 & 0.763
\end{tabular} & $\begin{array}{lllll}190 & 0.768 & 0.807\end{array}$ & \begin{tabular}{l|lll|}
173 & 0.708 & 0.776
\end{tabular} & 1900.7350 .755 & 1890.759 & 1770.901 \\
\hline 1900.7470 .780 & $\begin{array}{llll}190 & 0.741 & 0.763 \\
\end{array}$ & \begin{tabular}{l|lll}
181 & 0.754 & 0.807
\end{tabular} & $\begin{array}{llll}190 & 0.746 & 0.778\end{array}$ & 1890.761 & 1700.764 & 1700.933 \\
\hline 2020.848 & 2020.819 & 2020.877 & 2020.830 & $\begin{array}{lll}202 & 0.809\end{array}$ & 2040.8000 .801 & 2041.1540 .981 \\
\hline 1970.855 & \begin{tabular}{l|lll}
204 & 0.852 & 0.834
\end{tabular} & 1970.881 & 2010.840 & 2040.7890 .810 & 1980.8280 .809 & 1981.2131 .094 \\
\hline 2040.8800 .858 & 1970.835 & 2040.9110 .885 & 2040.8520 .849 & 2010.810 & 2030.8410 .812 & 2031.2301 .100 \\
\hline 2000.865 & 2000.836 & 2000.896 & 2030.8870 .859 & 1970.811 & \begin{tabular}{l|l|}
201 & 0.821
\end{tabular} & 2021.164 \\
\hline 2010.867 & 2010.839 & 2010.896 & 1980.8800 .860 & 2000.819 & $\begin{array}{ll}202 & 0.823\end{array}$ & 2011.172 \\
\hline 1960.873 & 1990.849 & $\begin{array}{llll}198 & 0.914 & 0.899\end{array}$ & 2000.864 & $\begin{array}{lllll}198 & 0.824 & 0.828\end{array}$ & $\begin{array}{lll}200 & 0.828\end{array}$ & 1971.194 \\
\hline 1990.877 & 1960.851 & 1960.901 & 1970.879 & $\begin{array}{llll}203 & 0.847 & 0.830\end{array}$ & 1970.829 & 2001.210 \\
\hline 1980.8830 .882 & $1980.854 \quad 0.853$ & \begin{tabular}{l|lll}
203 & 0.930 & 0.903
\end{tabular} & 1990.883 & 1990.847 & 2050.8660 .842 & 1961.229 \\
\hline 2030.8980 .885 & \begin{tabular}{l|ll}
203 & 0.870 & 0.855
\end{tabular} & 1990.907 & 1960.894 & 1960.849 & 1990.848 & 1991.230 \\
\hline 1950.895 & 1950.869 & 1950.927 & 1940.899 & 1950.859 & 1960.860 & 2051.2451 .254 \\
\hline 1940.903 & 1940.877 & 1940.935 & 1950.900 & 2050.8710 .865 & 1950.863 & 1941.261 \\
\hline 2050.9130 .922 & 2050.8860 .891 & 2050.9450 .944 & 2050.9090 .904 & 1940.886 & 1940.899 & 1951.284 \\
\hline 2081.0481 .029 & 2080.9860 .988 & 2081.0921 .073 & 2081.0441 .004 & 2080.9520 .955 & 2080.9660 .939 & 2081.6771 .661 \\
\hline 2071.0511 .042 & 2070.9931 .001 & 2071.0941 .089 & 2071.0371 .024 & 2070.9540 .965 & 2070.9710 .972 & 2071.6831 .741 \\
\hline 2061.0791 .074 & 2061.0181 .027 & 2061.1251 .111 & 2061.0801 .048 & 2060.9970 .997 & 2060.9991 .010 & 2061.7342 .091 \\
\hline 2091.2631 .219 & 2091.1391 .171 & 2091.3251 .290 & $2091.281 \quad 1.282$ & 2091.1951 .196 & 2091.2291 .281 & 2092.4682 .540 \\
\hline \multicolumn{7}{|l|}{ RT } \\
\hline$(\mathrm{min}) \mathrm{DB}-1$ & DB-5 & HT-5 & DB-17 & DB-XLB & HT-8 & CP-Sil19 \\
\hline 4721.46 & 25.81 & 21.59 & 27.92 & 31.29 & 35.18 & 32.23 \\
\hline 18349.82 & 56.72 & 51.00 & 62.63 & 64.67 & 69.69 & 70.25 \\
\hline
\end{tabular}

GC-RRTs for all peaks were calculated against the sum of the retention times (RTs) of BDEs 47 and 183. REF: reference value; PRE: predicted value. PRESS $($ DB-1 $)=0.00053$; PRESS $($ DB- $)=0.00049$; PRESS $($ HT- $)=0.00092 ;$ PRESS $($ DB-17 $)=0.00074 ;$ PRESS $($ DB-XLB $)=0.00040 ;$ PRESS $($ HT-8 $)=0.00042 ;$ PRESS $($ CP-Sil 19) $=0.0045$. PRESS:

Predicted error sum of squares.

${ }^{\mathrm{a}}$ Italic is the test set included $26 \mathrm{BDE}$ congeners,

${ }^{b}$ The bold is predicted test for 83 BDE congeners whichexperimental RRTs were not currently available. For predicted test of $83 \mathrm{BDE}$ congeners, BDE congeners were considered to co-elute if the difference between their predicted RRTs and experimental RRTs for training and test sets was $<0.001$ for peaks eluting before BDE47, and $\leq 0.002$ for peaks eluting after BDE 47 .

stitutional, topological, geometrical, electrostatic, and semiempirical quantum.

GC-RRTs of 17 BDEs $(4,20,27,58,72,76,81,98,103,115$, $121,127,158,160,182,185$, and 203) are included in both our test sets and predicted sets by models in ref. [21] on DB-5 stationary phase. Fig. 3 compares experimental and predicted GC-RRT of our work and Rayne's work. The curving line is experimental data (ref. [21]) versus predicted GC-RRT of Rayne's work (ref. [20]) and the linear line is experimental data (ref. [21]) versus predicted GC-RRT of this work. The fitting results show the high agreement between the different temperature programmes.

\subsection{Comparison in retention behavior between PBDEs and $\mathrm{PCBS}$}

Based on the retention database for $\mathrm{PCBs}$ built by $\mathrm{Chu}$ and Hong [29] and retention database for PBDEs expanded in our study (ref. [21] RRTs + Predicted RRTs), the relation of retention behavior on DB-5, DB-17 and DB-XLB column between these two classes of pollutants were studied. The best correlation coefficient for DB-5 was 0.965 , for DB-17 was 0.957 , and for DB-XLB was 0.960 (Fig. 4). But in fact, there were some differences that reflect the retention behavior between PCBs and PBDEs. Calculational results show that the molecules of PBDEs are not coplanar at the conformation of their lowest energy [30]. There were also some other differences; for example, our models included the descriptor "minimum electrophilic reaction index for a $\mathrm{O}$ atom", which indicated that $\mathrm{O}$ atom of PBDEs would be an important factor that reflect the elution order comparing to PCBs.

The retention time of PBDEs generally increases with the bromine substitution, with little crossover among homologs when a nonpolar stationary phase is used. In other words, PBDE congeners with $n+1$ bromines usually elute after those with $n$ bromines, with only a few exceptions. This is in contrast to PCBs 
whose retention time order is more chaotic in regard to the degree of chlorination. This may be related to the effect of molecular planarity, which is possible for some PCB congeners but not others in the same homolog, on the chromatographic retention. Due to the steric and probably also electronic effect of the ether linkage, it is impossible for PBDEs to be planar, regardless of the number and the positions of bromines. Although the angle between the two phenyl rings at optimal conformation does differ among structural isomers depending on bromine positions [31], the effect of these difference on GC retention may be much less than the number of bromines in the molecule.

\subsection{Model application}

By using the models built, the RRTs of remaining $83 \mathrm{BDE}$ congeners for which experimental RRTs were not currently available were predicted, and the results were summarized in Table 3. The congeners enclosed in a box were considered coeluting (RRTs differ by less than 0.001 before BDE47, less than 0.002 after BDE47) on specified columns.

The predicted RRTs in this work were plotted in Fig. 5 against model predicted RRTs in the ref. [20]. The plot shows a good general agreement of these two models. For the whole data matrix, the fitting curve followed the natural logarithm function due, again, to the different oven temperature programming and other operational parameters. However, for the congeners that have the same number of bromine atom, the fitting relationship is linear.

Relative retention varies with the changes in instrument configurations, column specifications, and operation conditions. In applying the established RRT models to identify the unknowns, optimal predictability is obtained only when the instrument con-
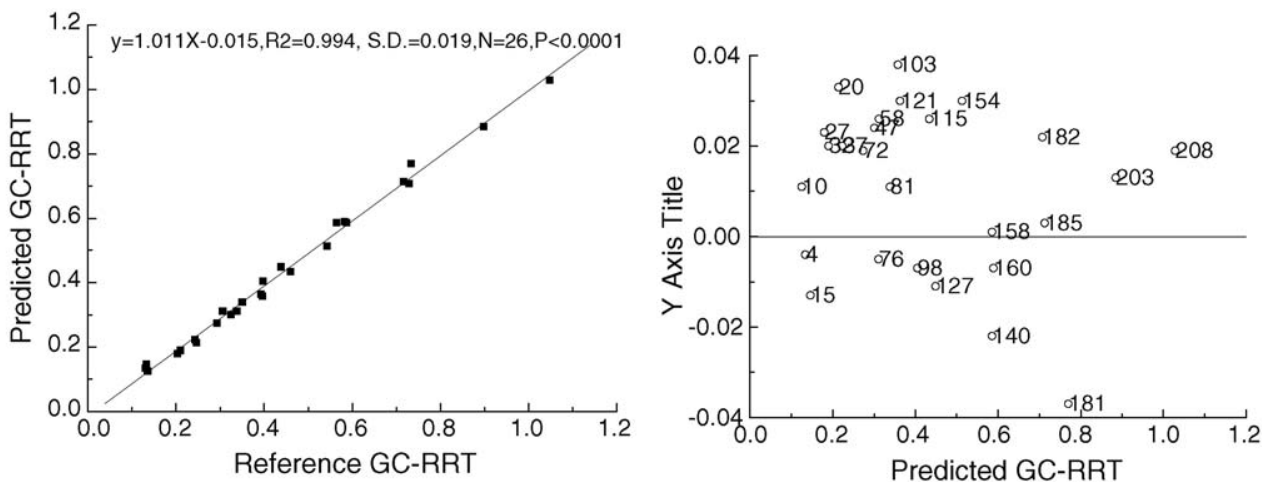

DB-1
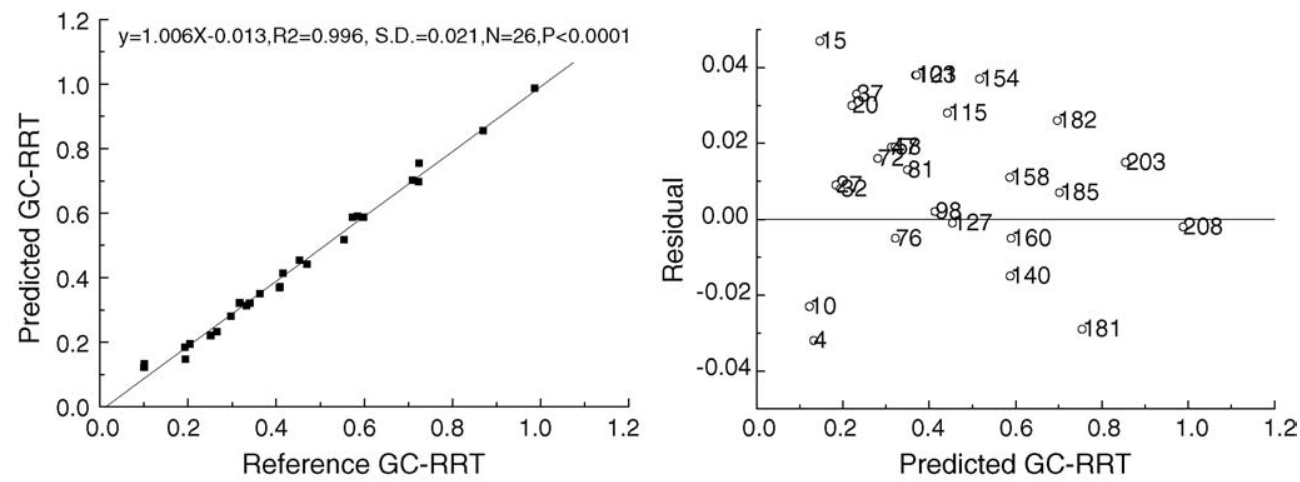

DB-5
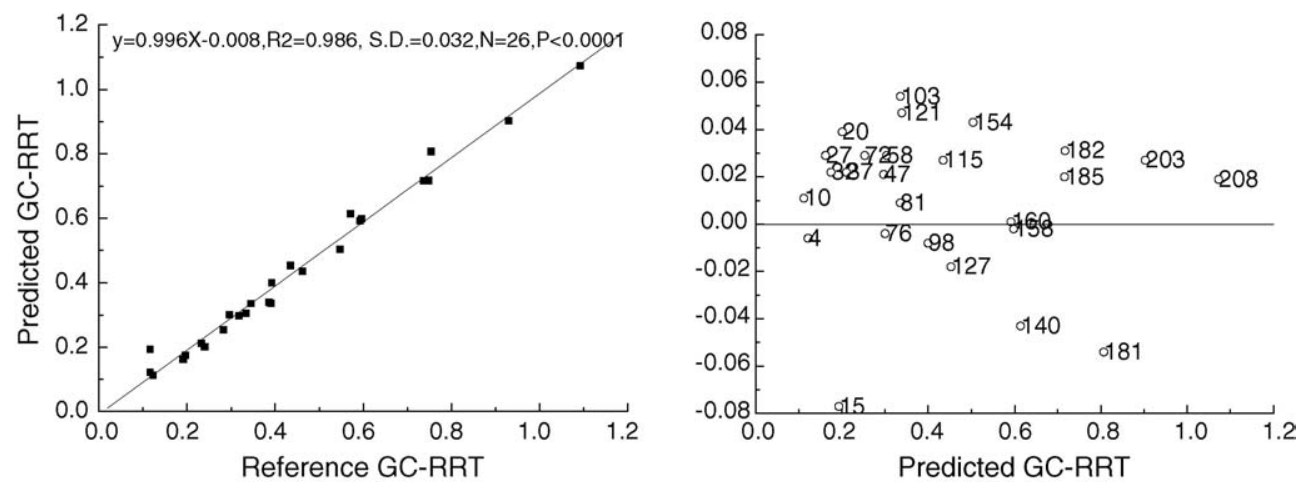

HT-5

Fig. 2. Reference vs. predicted RRTs of 26 PBDE congeners in the test sets on different columns and distribution of residuals. 

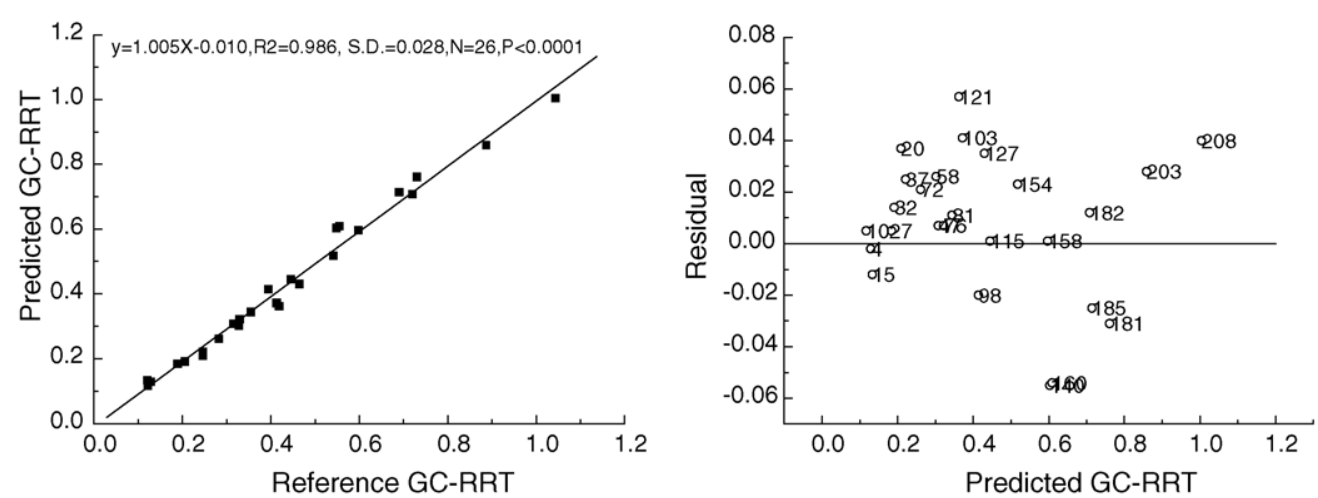

DB-17
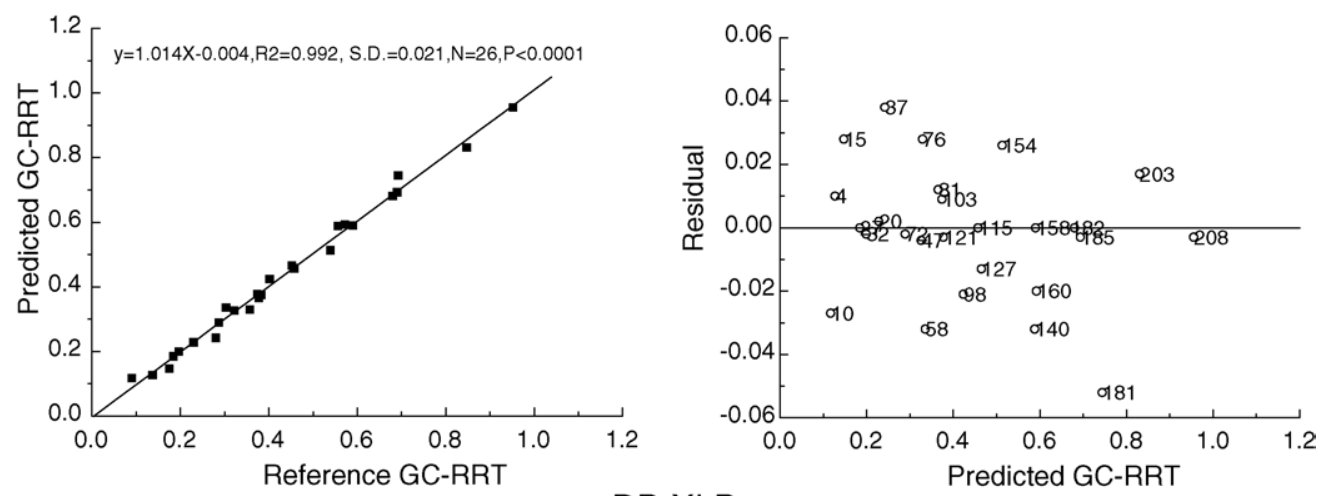

\section{DB-XLB}
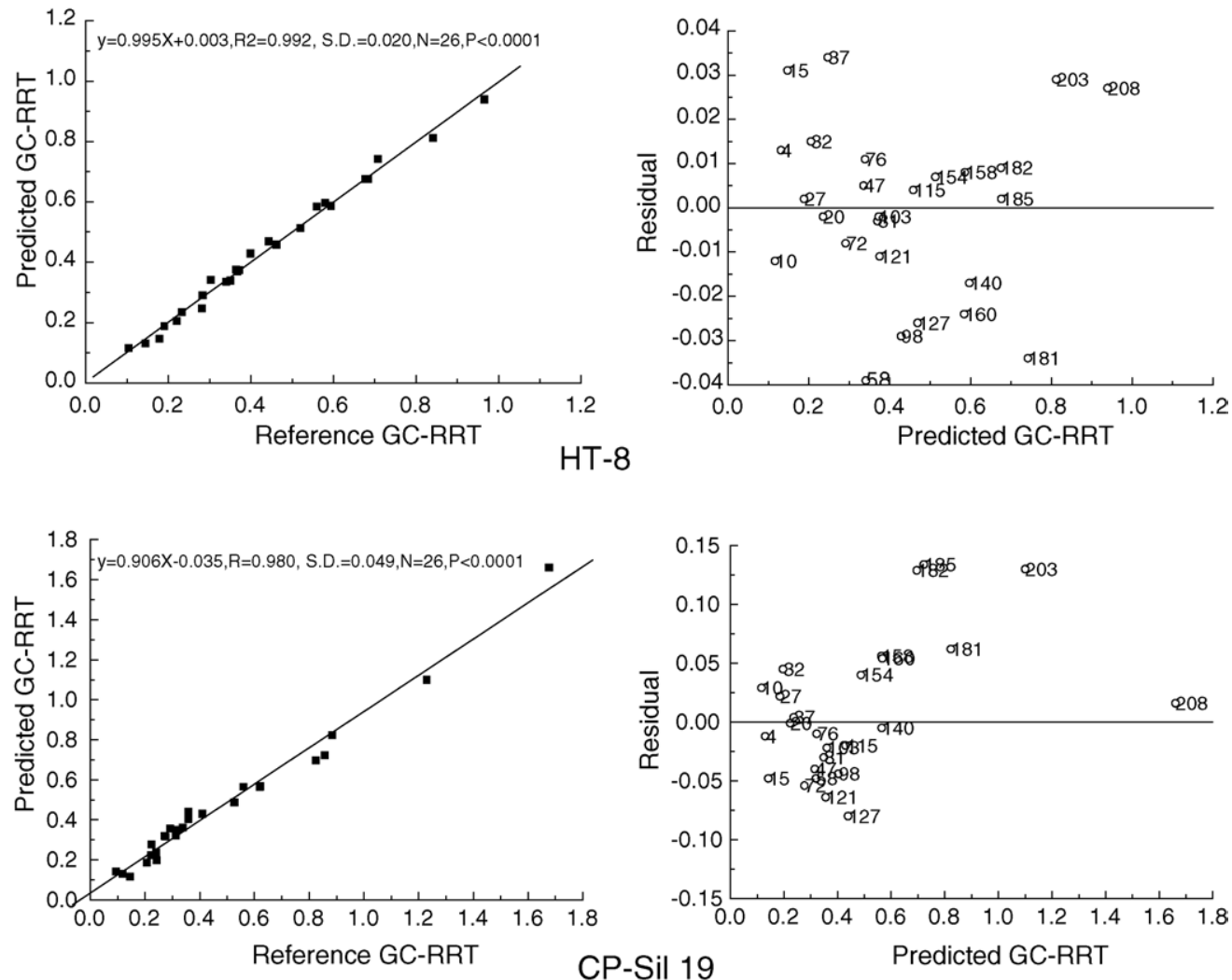

Fig. 2. (Continued). 


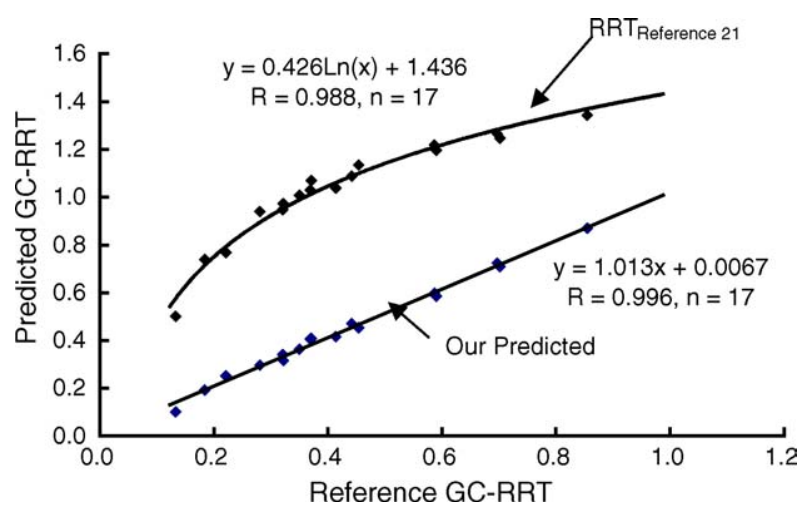

Fig. 3. Comparison the RRTs of 17 PBDE congeners in the test set on DB-5 predicted by the models presented in ref. [22] and this work.
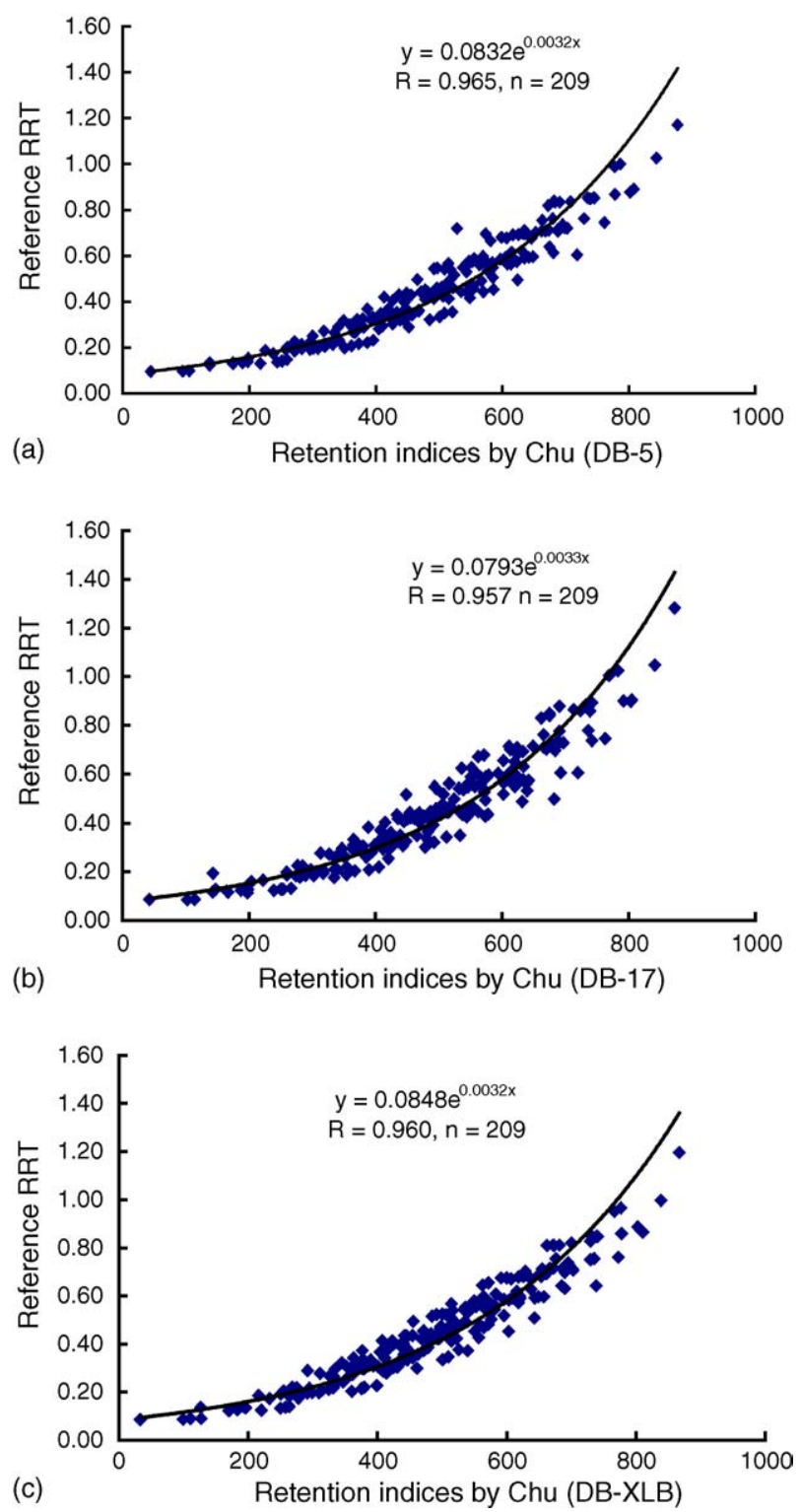

Fig. 4. Retention indices based on the retention database for PCBs (ref. [32]) vs. retention database for PBDEs (database of ref. RRTs + database of predicted RRTs by our models) on DB-5, DB-17, and DB-XLB column. (a) DB-5; (b) DB-17; and (c) DB-XLB.

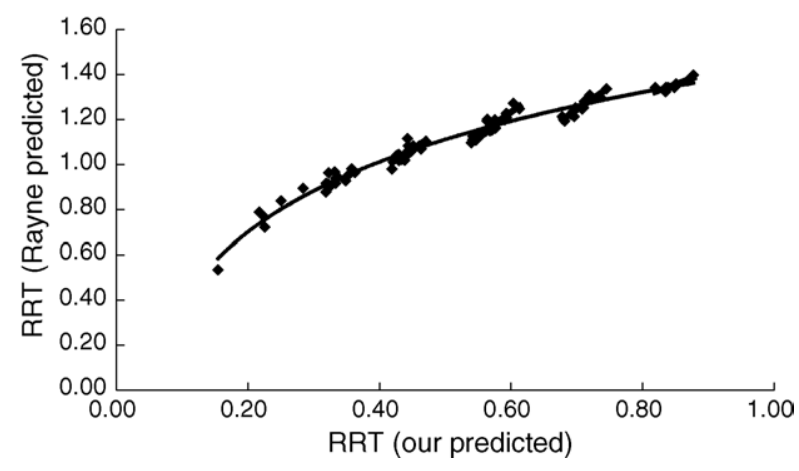

Fig. 5. Comparison between the RRTs of the 83 PBDE congeners, for which ref. RRTs are unavailable, on DB-5 predicted by the models presented in ref. [22] and this work.

figuration and operation conditions are exactly the same as those used in the model development. For this reason, models for chromatographic retention prediction were often built using either isothermal or linear temperature programs [32]. However, it is practically impossible to obtain RRTs for PBDEs with isothermal or linear temperature programs, as explained in details by Rayne and Ikonomou [20].

In this work, the predictability of our models was assessed using analytical results obtained under different instrument configuration and/or operation conditions. The reference RRTs used in this work were obtained by Korytár et al. [21] with the use of multi-linear temperature programs (starting at $90^{\circ} \mathrm{C}$ for $2 \mathrm{~min} ; 30^{\circ} \mathrm{C} / \mathrm{min}$ to $200^{\circ} \mathrm{C} ; 1.5^{\circ} \mathrm{C} / \mathrm{min}$ to $325^{\circ} \mathrm{C}$ ). We used two DB-5MS columns with the same diameter and film thickness as those used by Korytár et al. [21] to obtain the RRTs of selected PBDE congeners. Two runs were made with 12 and $30 \mathrm{~m}$ columns, respectively, on different instruments with very different temperature programs, as specified in Section 2. In addition, a set of retention times $(N=13)$ obtained by Agilent on a DB-XLB column $(30 \mathrm{~m} \times 0.25 \mathrm{~mm}$ i.d. $\times 0.1 \mu \mathrm{m}$ film thickness, constant flow at $1.2 \mathrm{~mL} \mathrm{~min}^{-1}$ ) coupled with an ICPMS [33] was also transformed into RRTs, which was then used as an externally obtained dataset to examine the model performance. Although the three curves in Fig. 6 are different in shape, all of them are smooth with $R>0.995$, showing strong correlations between the two datasets. Reversal of the elution order, which was observed for several pairs of PCB congeners when oven temperature was increased at different rates [29], was not observed in this work with the limited numbers of PBDEs involved.

These observations point to the possibility of potentially high accurate predictions of RRTs using the established models. While the curvatures in Fig. 6 are most likely attributed to the differences in instrument configuration and operational parameters, the smoothness of the curves may imply that the selected PBDE congeners respond to the changes in GC parameters primarily in a systematic manner with a common mechanism. Regression equations shown in Fig. 6 can be used to "translate" the experimental RRTs into the RRTs to be used in the model, from which the unknown congeners can be identified as long as their molecular descriptors are available. Until 

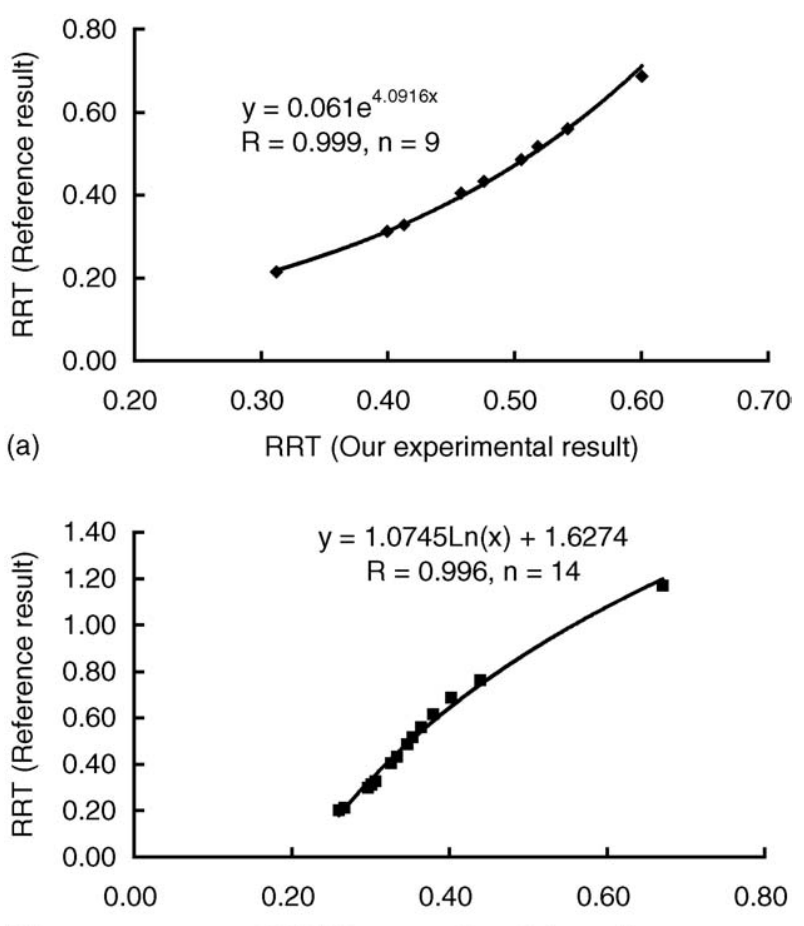

(b) RRT (Our experimental result)

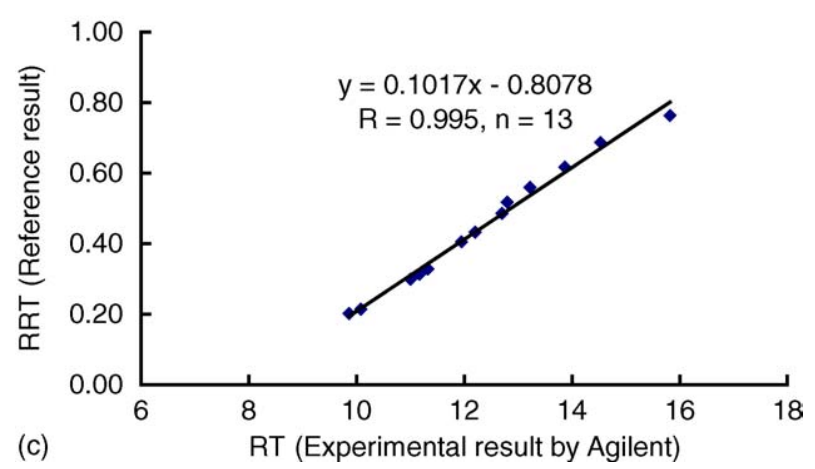

Fig. 6. Correlations of GC-RRTs on a DB-5 column in ref. [23] and in this work using (a) HRGC-LRMS, (b) HRGC/HRMS, and (c) Db-XLB column using GC-ICP-MS.

an approach, which is independent of instrument configuration and operation conditions, can be established, predictions of RRTs must take into consideration the impact of these variables. Simply matching the RRTs obtained with those in the reference dataset may result in faulty identification of the peaks.

\section{Conclusions}

Based on an experimental retention-time database of 126 PBDE congeners for seven GC columns [21], quantitative structural-retention relationship models were built to study the retention behavior on these GC columns by the heuristic method of CODESSA. The results show that the models have strong predictive abilities. The intermolecular interactions betweens PBDEs and the stationary phase are related to the degree of branching of the molecules, which can be represented by Wiener or Randic index. Further, using these models, RRTs of the remaining 83 PBDEs for which retention times are currently not available, were predicted. Although nonlinear, strong correlations were obtained between RRTs obtained using different GC configurations and under different operation conditions. Once established, the correlation equations can be used to predict the chemical identities of unknown peaks.

\section{Acknowledgements}

Financial support of this work was provided by the National Basic Research Program of China (2003CB415001). The authors would like to thank Professor Man-cang Liu and his group at the Department of Chemistry, Lanzhou University, China for the valuable discussions.

\section{References}

[1] R. Renner, Environ. Sci. Technol. 34 (2000) 223A.

[2] R.C. Hale, M.J. La Guardia, E.P. Harvey, T.M. Mainor, W.H. Duff, M.O. Gaylor, Environ. Sci. Technol. 35 (2002) 4585.

[3] A. Covaci, A. Gheorghe, S. Voorspoels, J. Maervoet, E. Steen Redeker, R. Blust, P. Schepens, Environ. Int. 31 (2005) 367.

[4] K. Booij, B.N. Zegers, J.P. Boon, Chemosphere 46 (2002) 683.

[5] K. Norén, D. Meironyte, Chemosphere 40 (2000) 1111.

[6] I.A.T.M. Meerts, J.J. van Zanden, E.A.C. Luijks, I. van Leeuwen-Bol, G. Marsh, E. Jakobsson, A. Bergman, A. Brouwer, Toxicol. Sci. 56 (2000) 95.

[7] WHO, 1994. Environmental health criteria 162: Brominated diphenyl ethers. World Health Organisation, Geneva, p. 31.

[8] J.B. Cruz, C.T. Jafvert, I. Hua, Environ. Sci. Technol. 38 (2004) 4149.

[9] Y.S. Keum, Q.X. Li, Environ. Sci. Technol. 39 (2005) 2280.

[10] M. Alaee, D.B. Sergeant, M.G. Ikonomou, J.M. Luross, Chemosphere 44 (2001) 1489.

[11] P.S. Haglund, D.R. Zook, H.R. Buser, J.W. Hu, Environ. Sci. Technol. 31 (1997) 3281.

[12] E. Eljarrat, S. Lacorte, D.J. Barcelo, J. Mass Spectrom. 37 (2002) 76.

[13] T. Körtvélyesi, M. Görgényi, K. Héberger, Anal. Chim. Acta 428 (2001) 73.

[14] V.S. Ong, R.A. Hites, Anal. Chem. 63 (1991) 2829.

[15] T. Nevalainen, J. Koistinen, P. Nurmela, Environ. Sci. Technol. 28 (1994) 1341.

[16] M.D. Hale, F.D. Hileman, T. Mazer, T.L. Shell, R.W. Noble, J.J. Brooks, Anal. Chem. 57 (1985) 640.

[17] R. Hackenberg, A. Schutz, K. Ballschmiter, Environ. Sci. Technol. 37 (2003) 2274.

[18] M.G. Ikonomou, S. Rayne, Anal. Chem. 74 (2002) 5263.

[19] S. Rayne, M.G. Ikonomou, Anal. Chem. 75 (2003) 1049.

[20] S. Rayne, M.G. Ikonomou, J. Chromatogr. A 1016 (2003) 235.

[21] P. Korytár, A. Covaci, J. de Boer, A. Gelbin, U.A.Th. Brinkman, J. Chromatogr. A 1065 (2005) 239.

[22] J.P.P. Stewart, MOPAC, v.6.0, 1989. Quantum Chemistry Program Exchange, QCPE, No. 455; Indiana University, Bloomington, IN.

[23] A.R. Katritzky, V. Lobanov, M. Karelson, Chem. Soc. Rev. 24 (1995) 279.

[24] A.R. Katritzky, V.S. Lobanov, M. Karelson, Comprehensive descriptors for structural and statistical analysis, Reference Manual, Version 2.13, 1995-1997.

[25] H. Wiener, J. Am. Chem. Soc. 69 (1947) 17.

[26] D.H. Rouvray, Sci. Am. 254 (986) 40. 
[27] M. Randic, J. Am. Chem. Soc. 97 (1975) 6609.

[28] Y.W. Wang, X.J. Yao, X.Y. Zhang, R.S. Zhang, M.C. Liu, Z.D. Hu, B.T. Fan, Talanta 57 (2002) 641.

[29] S.G. Chu, C.-S. Hong, Anal. Chem. 76 (2004) 5486.

[30] Y.W. Wang, H.X. Liu, C.Y. Zhao, H.X. Liu, Z.W. Cai, G.B. Jiang, Environ. Sci. Technol. 39 (2005) 4961.
[31] D.Y. Zeng, D. Tsukada, J.A. Noblet, J. Peng, J. Chromatogr. A 1066 (2005) 165

[32] E. Kovàts, Helv. Chim. Acta 41 (1958) 1915.

[33] GC Analysis of polybrominated flame retardants, Application, 2003, http://www.getech.com.tw/upload/113/\%7BBD744F28-28EE-4442ACA3-4C12843C15F7\%7D_EPA-005.pdf. 